\title{
Ischemic post-conditioning attenuates acute lung injury induced by intestinal ischemia-reperfusion in mice: role of Nrf2
}

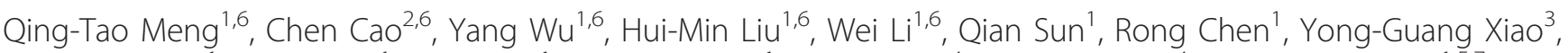 \\ Ling-Hua Tang ${ }^{1}$, Ying Jiang ${ }^{1}$, Yan Leng ${ }^{1}$, Shao-Qing Lei ${ }^{1}$, Chris C Lee ${ }^{4}$, Devin M Barry ${ }^{4}$, Xiangdong Chen ${ }^{1,5,7}$ and \\ Zhong-Yuan Xia ${ }^{1,7}$
}

Intestinal ischemic post-conditioning (IPo) protects against lung injury induced by intestinal ischemia-reperfusion (IIR) partly through promotion of expression and function of heme oxygenase-1 (HO-1). NF-E2-related factor-2 (Nrf2) is a key transcription factor that interacts with HO-1 and regulates antioxidant defense. However, the role of Nrf2 in IPo protection of IIR-induced pulmonary injury is not completely understood. Here we show that IPo significantly attenuated IIR-induced lung injury and suppressed oxidative stress and systemic inflammatory responses. IPo also increased the expression of both Nrf2 and HO-1. Consistently, the beneficial effects of IPo were abolished by ATRA and Brusatol, potent inhibitors of Nrf2. Moreover, the Nrf2 agonist t-BHQ showed similar activity as IPo. Taken together, our data suggest that Nrf2 activity, along with HO-1, plays an important role in the protective effects of IPo against IIR-induced acute lung injury.

Laboratory Investigation (2016) 96, 1087-1104; doi:10.1038/labinvest.2016.87; published online 8 August 2016

Intestinal ischemia-reperfusion (IIR) injury is one of the most serious and common clinical events in disorders such as hemorrhagic shock, intestinal obstruction, intussusception, neonatal necrotizing enterocolitis, and geriatric mesenteric insufficiency. ${ }^{1}$ Barrier dysfunction caused by IIR eventually results in translocation of bacteria and toxins through a leaky gut mucosa, and amplifies or perpetuates systemic inflammation and oxidative stress, leading to multiple organ dysfunction syndrome (MODS) and death in critically ill patients. ${ }^{2,3}$ The lungs are often the most frequently affected organs by IIR, and in some instances it leads to the development of acute lung injury (ALI) and acute respiratory distress syndrome (ARDS). ${ }^{4}$ Despite numerous attempts to improve the outcome in patients with ARDS, a mortality rate around $50 \%$ is still encountered. ${ }^{5}$

Ischemic post-conditioning (IPo) has been demonstrated to be beneficial following ischemia/reperfusion (IR) injury in the heart, brain, liver, kidney, and spinal cord, ${ }^{6}$ and its mechanical interventions against reperfusion injury relate to multiple and interacting components in endogenous protective mechanisms. Previous studies have shown that IPo can also attenuate lung injury induced by IIR, in part, through the activation of heme oxygenase-1 (HO-1). ${ }^{7}$ However, the underlying molecular mechanisms, by which IPo regulates HO-1 expression or activity, remain poorly understood.

Nrf2 belongs to the Cap'n'collar/basic region leucine zipper transcription factor family, and serves as a pleiotropic regulator in cell survival mechanisms. ${ }^{8}$ The conditions of oxidative or xenobiotic stress lead to dissociation of Nrf2 from Keap1 translocation to the nucleus, and sequentially enhanced transcription of target antioxidant/detoxifying genes via the antioxidant response element (ARE). ${ }^{9}$ Recent studies suggest that Nrf2 plays a protective role against lung injury induced by oxidative stress, including hyperoxia, mechanical ventilation, and cigarette smoke, possibly through transcriptional activation of antioxidant defense enzymes such as HO- 1 in the lung. ${ }^{10}$ These findings

'Department of Anesthesiology, Renmin Hospital of Wuhan University, Wuhan, China; ${ }^{2}$ The Medical Department, the 3rd Hospital of Wuhan, Wuhan, China; ${ }^{3}$ Department of Thoracic Surgery, Renmin Hospital of Wuhan University, Wuhan, China; ${ }^{4}$ Department of Anesthesiology, Washington University School of Medicine, St Louis, MO, USA and ${ }^{5}$ Department of Anesthesiology, Union Hospital, Tongji Medical College, Huazhong University of Science and Technology, Wuhan, China

Correspondence: Dr Z-Y Xia, PhD, Department of Anesthesiology, Renmin Hospital of Wuhan University, Jiefang Road 238, Wuhan 430060, China.

E-mail: xiazhongyuanmz@hotmail.com

${ }^{6}$ These authors contributed equally to this work.

${ }^{7} Z-Y X$ and $X C$ share senior authorship.

Q-TM, Z-YX and XC designed research; Q-TM, YJ, QS, H-ML and RC performed the majority of experiments; Z-YX provided vital reagents and analytical tools and was also involved in editing the manuscript; Q-TM and CC analyzed data; Q-TM, YW and Y-GX wrote the paper. Illustrations and proofreading was performed by Z-YX, WL, S-QL, CCL and DMB.

Received 7 April 2016; revised 3 July 2016; accepted 3 July 2016 
a

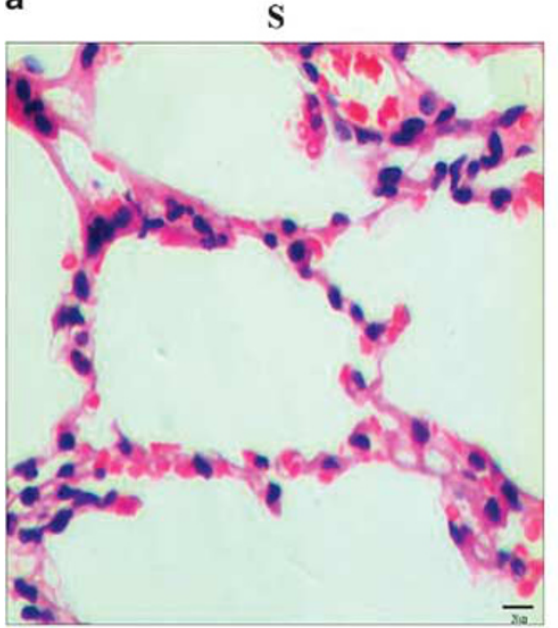

b

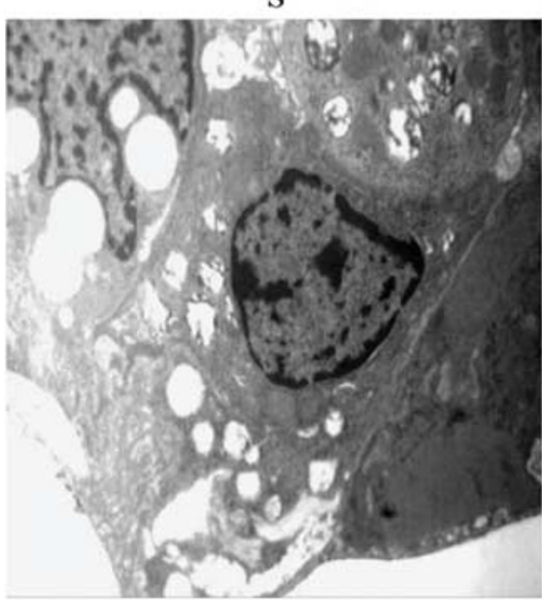

IIR

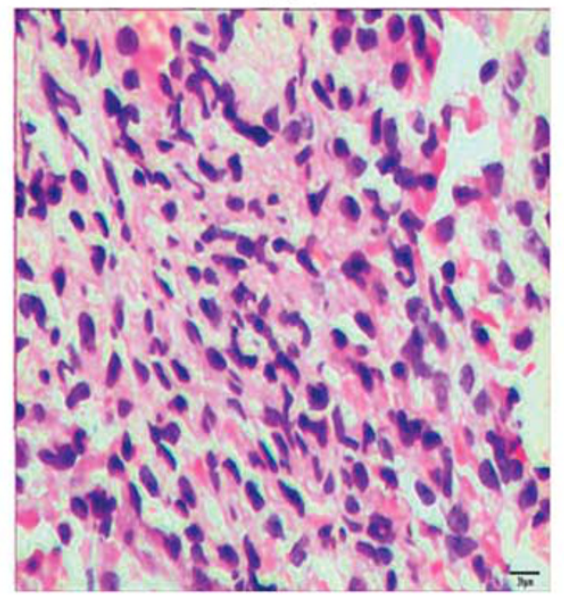

IIR

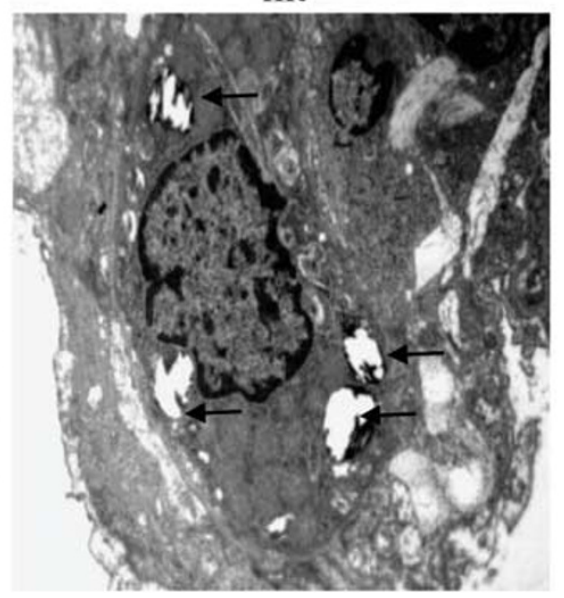

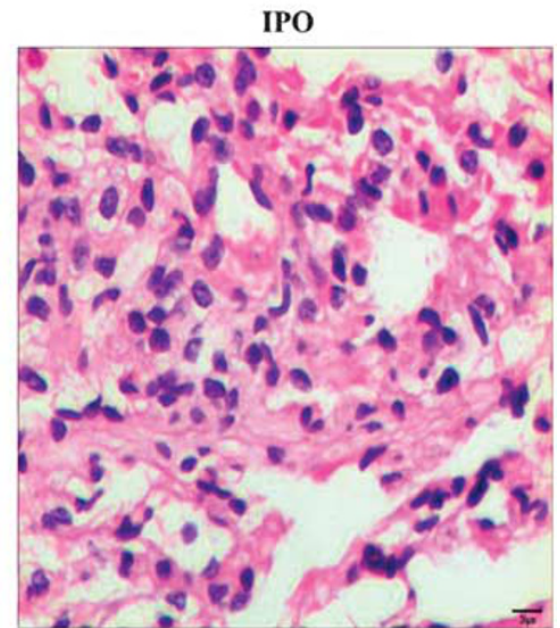

IPO

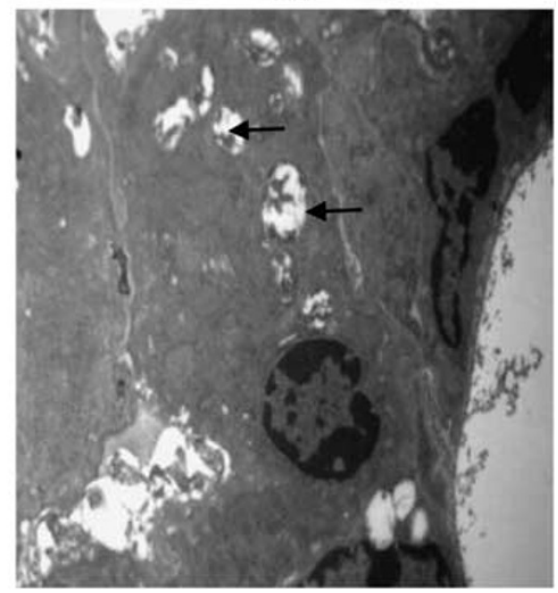

Figure 1 The protective effect of IPo on IIR-induced Lung injury. (a) Histopathologic changes of mice lung under a light microscope ( $\times 400$, H\&E staining). Forty-five minutes of superior mesenteric artery occlusion followed by $120 \mathrm{~min}$ reperfusion caused an excessive alveolar interstitial edema, telangiectasis, hemorrhage, thickening of the alveolar wall, and infiltration of inflammatory cells into the interstitial and alveolar spaces (IIR). These features were dramatically ameliorated after treated with IPo. There were minimal edema, hemorrhage, and inflammatory after IPo. Scale bar, $20 \mu \mathrm{m}$. (b) The pulmonary electron microscopic evaluation. Forty-five minutes of superior mesenteric artery occlusion followed by 120 min reperfusion caused a swollen of the basement membranes of alveolar epithelium and capillary endothelium, and reduced or even vanished the electronic density. With the swollen of the mitochondria, the empty of osmiophilic lamellar bodies, and the lodged and disappeared cellar ridge caused by IIR were observed in type II alveolar cells. Moreover, the conjunctions between the alveolar epithelial cells and the capillary endothelial cells were injured with gaps (IIR), which are significantly different from control (group S, S). These features dramatically diminished after treating with IPo (IPO). Scale bar, $2000 \mathrm{~nm}$. (c) Summary of ALI scores in different groups. ALI scores significantly increased after IIR injury. However, IPo treatment can significantly decrease the ALI induced by IIR injury. Arrows indicate. (d, e) The effects of IPo on the pulmonary microvascular permeability (e) and lung tissue wet/dry weight ratios (f) in lung tissue of mouse. Data presented as mean \pm s.d., $n=10$. ${ }^{*} P<0.05$ vs $S$ group, ${ }^{\#} P<0.05$ vs IIR group, one-way ANOVA and Tukey's post hoc test.

suggest that Nrf2 may be the key modulator of lung injury induced by IIR, and Nrf2 might be a potential therapeutic target for lung injury after IIR challenge. The purpose of this study was to investigate the actions of Nrf2 in IPo-mediated protection against pulmonary injury induced by IIR and to examine the expression of Nrf2 and HO-1 in IPo-mediated protection.

\section{MATERIALS AND METHODS}

\section{Animals and Reagents}

This study complied with the Guide for the Care and Use of Laboratory Animals by the National Institutes of Health $\mathrm{NIH}$
Publication No. 80-23) and was approved by Bioethics Committee of Renmin Hospital of Wuhan University (Wuhan, China). This study was performed in the animal center of Renmin Hospital of Wuhan University from November 2010 to May 2012. Adult male C57BL/6 J mice (Hunan Slac JD Laboratory Animal, Hunan, China) weighing $25 \pm 3 \mathrm{~g}$ were housed in individual cages in a temperaturecontrolled room with alternating 12-h light/dark cycles and acclimated for 2 weeks before experiments. All the animals were fasted $12 \mathrm{~h}$ before the experiments but had free access to water ad libitum. The glutathione peroxidase (GSH-Px), 

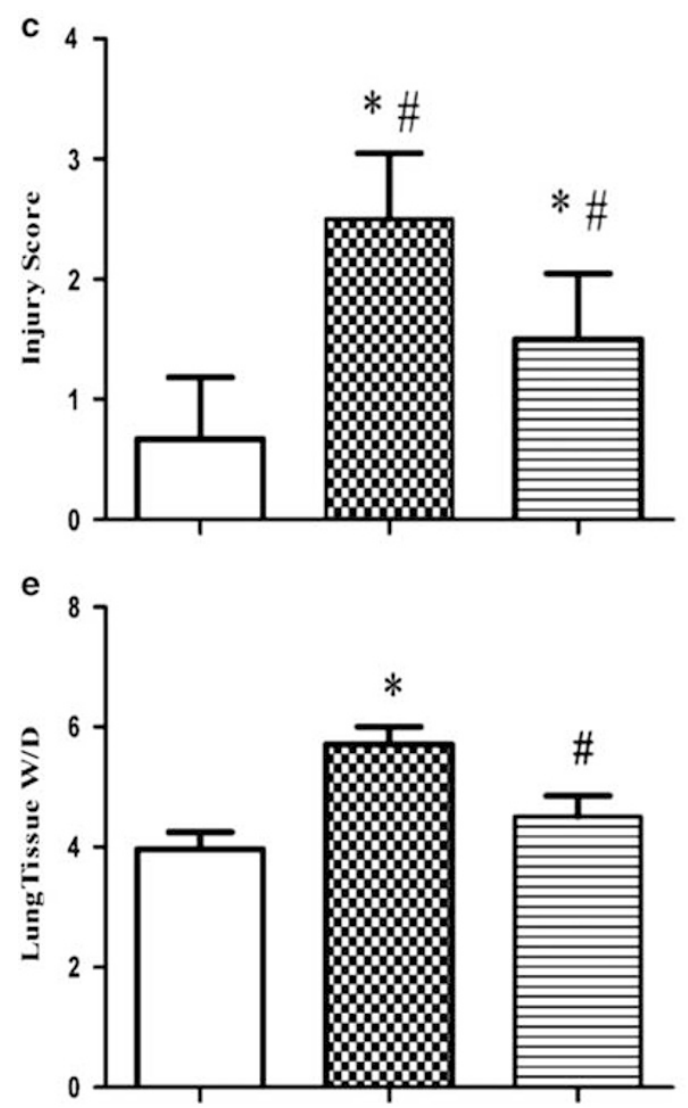

Figure 1 Continued.

superoxide dismutase (SOD), and malondialdehyde (MDA) assay kits were obtained from Nanjing Jiancheng Bioengineering Institute (Nanjing, China). Antibodies for Nrf2 and HO-1 were purchased from Santa Cruz Biotechnology (Santa Cruz, CA, USA). Tumor necrosis factor- $\alpha$ (TNF- $\alpha$ ), interleukin-6 (IL-6), and interleukin-10 (IL-10) ELISA kits specific for mouse cytokines were obtained from American R \& D (Minneapolis, MN, USA). Brusatol and all-trans-retinoic acid (ATRA), the specific antagonist of Nrf2, ${ }^{11,12}$ were purchased from Sigma-Aldrich (Shanghai, China). t-BHQ, the specific agonist of $\mathrm{Nrf2},{ }^{13}$ was purchased from Sigma-Aldrich. All other chemicals used were of the highest grade available commercially.

\section{Experimental Protocol and Intestinal Ischemia-Reperfusion Model}

The animals were randomly allocated into 12 groups as follows: (i) S group; (ii) IIR group (intestinal ischemia reperfusion group); (iii) IPo group (IIR+IPo); (iv)ATRA+S group; (v) ATRA+IIR group; (vi)ATRA+IPO group; (vii) Brusatol $+S$ group; (viii) Brusatol +IIR group; (ix) Brusatol $+\mathrm{IPO}$ group; $(\mathrm{x}) \mathrm{t}-\mathrm{BHQ}+\mathrm{S}$ group; (xi) $\mathrm{t}-\mathrm{BHQ}+\mathrm{IIR}$ group; and (xii) t-BHQ +IPO group. About $16.7 \mathrm{mg} / \mathrm{kg} \mathrm{t}-\mathrm{BHQ}$ diluted in $1 \%$ DMSO in phosphate-buffered saline (PBS) was injected i.p. every $8 \mathrm{~h}$ for 3 days. ${ }^{13}$ the ATRA group received

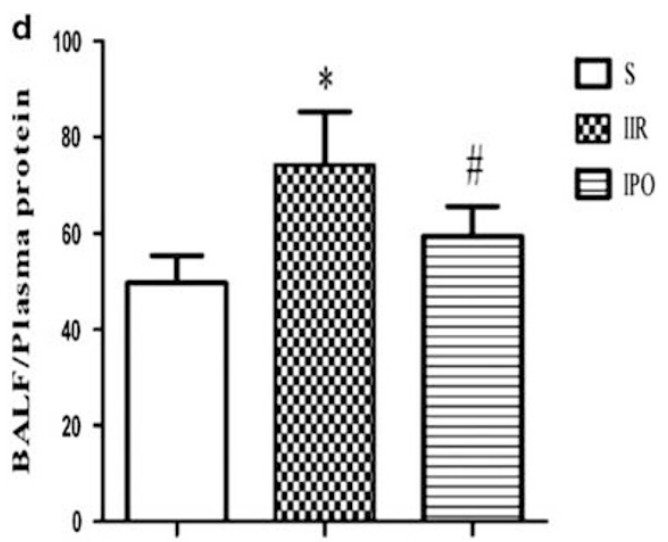

ATRA $(2 \mathrm{mg} / \mathrm{ml}$, dissolved in saline, $10 \mathrm{ml} / \mathrm{kg}$ intraperitoneal (i.p.) daily) for 2 weeks before the experiment. ${ }^{11}$ For Brusatol treatment, animals received Brusatol $(0.5 \mathrm{mg} / \mathrm{ml}$, diluted by $1 \%$ DMSO) intraperitoneal injection once per 2 days for 10 days before the experiment. ${ }^{12}$ T-BHQ-treated groups were injected i.p. with $\mathrm{t}-\mathrm{BHQ}$ at a dose of $16.7 \mathrm{mg} / \mathrm{kg}$ every $8 \mathrm{~h}$ for 3 days. ${ }^{13}$ After the treatment, the animals were anesthetized with sodium pentobarbital $(50 \mathrm{mg} / \mathrm{kg})$ intraperitoneally. Surgery was performed after loss of the blink and withdrawal reflexes. The mice were then placed in the supine position and allowed to breathe spontaneously. The IIR model was established by superior mesenteric artery (SMA) occlusion. ${ }^{14}$ And after laparotomy the small bowel was retracted to the left, and the SMA was temporarily occluded using a microvascular clip. Mice was subjected to ischemia $(45 \mathrm{~min})$ followed by 120 min reperfusion (I/R) via gently removing the clip and mice in the $S$ group underwent the same surgical manipulation except without SMA occlusion. IPo was induced by three cycles of reperfusion (30s) and re-occlusion (30s) before 120 min of reperfusion. ${ }^{15}$

\section{The Histopathology of Lung Tissue (Haematoxylin and Eosin Stain)}

The upper lobe of the left mice lungs was fixed in $10 \%$ buffered formalin, and $4 \mathrm{~mm}$ sections were prepared from 
a

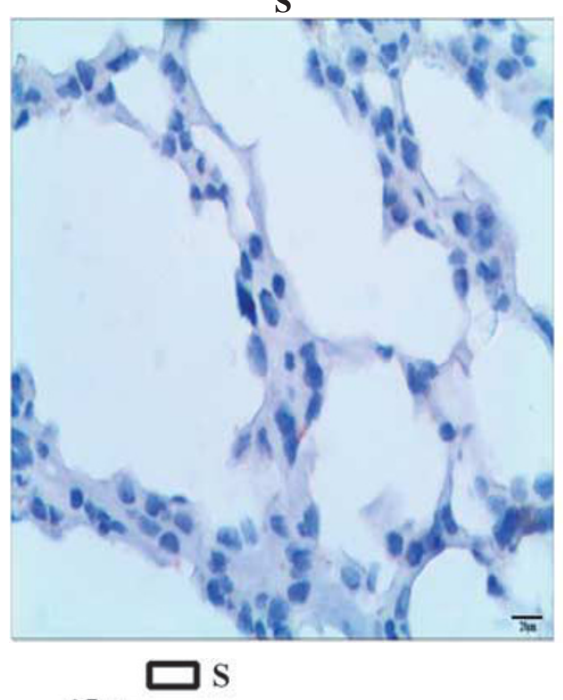

IR

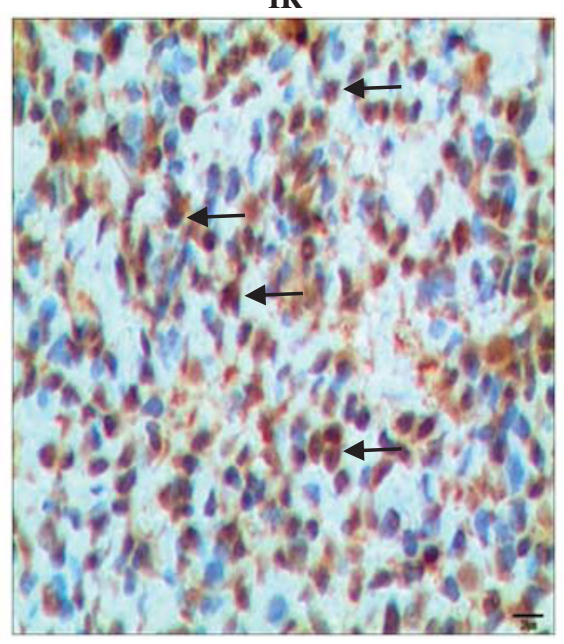

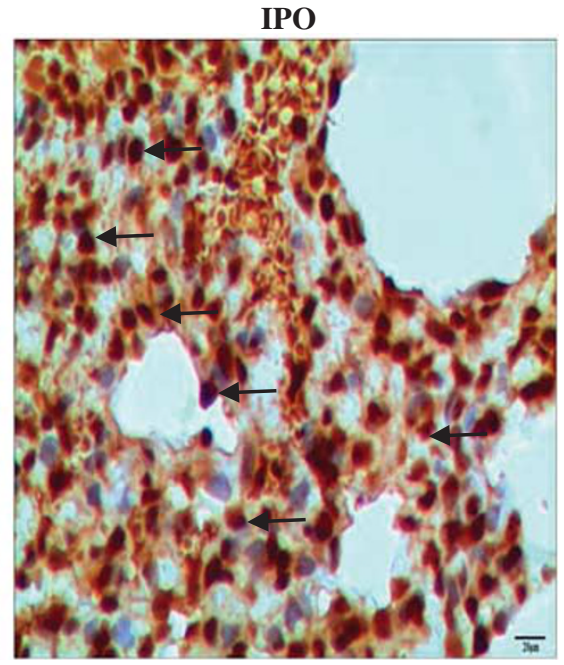

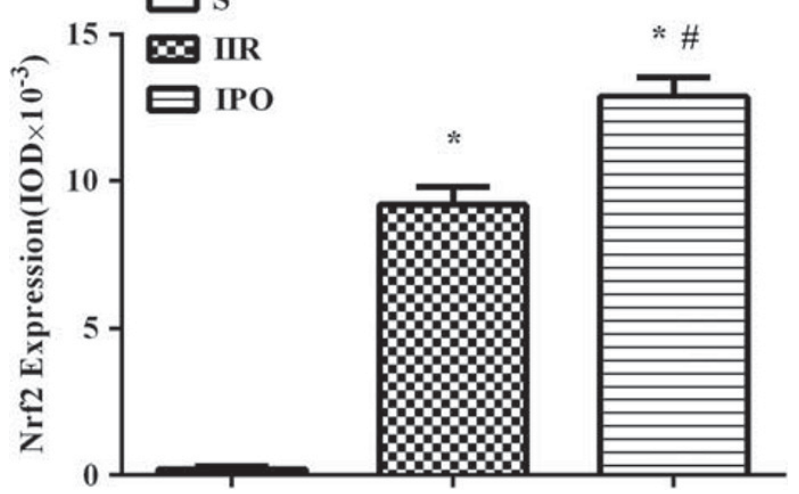

Figure 2 The expression of Nrf2 and HO-1 in lung tissue of mouse. (a, b). The expressions of Nrf2 (a) and HO-1 (b) were detected by immunohistochemistry with the Streptomyces avidin-peroxidase connection (SP) method. The expressions of Nrf2 and HO-1 mainly located in the cytoplasm and nucleus of bronchus smooth muscle cells, bronchus epithelial cells, vascular smooth muscle cell, and alveolar epithelial cells. Parts of lung tissue cells cytoplasm showed brownish yellow in the $S$ group (S). A large number of lung tissue cells cytoplasm and nucleus showed brownish yellow or dark brown in the IIR group (IIR), and a great number of lung tissue cells cytoplasm and nucleus showed brownish yellow or dark brown in the IPo group (IPO). Scale bar, $20 \mu \mathrm{m}$. Arrows indicate. (c, d) The expressions of Nrf2 and HO-1 in the lung were detected by western blotting in each group. Lamin-B detected as a loading control of Nrf2 (c) by western blot, and $\beta$-action detected as a loading control of HO-1 (d) by western blotting. Data presented as mean \pm s.d., $n=10 .{ }^{*} P<0.05$ vs $S$ group, ${ }^{\#} P<0.05$ vs IIR group, one-way ANOVA and Tukey's post hoc test.

the paraffin-embedded tissue. The histology of lungs was assessed by hematoxylin and eosin staining under light microscopy. At least two different sections of each specimen were examined.

ALI was evaluated by a pathologist initially unaware of the experimental groups (NZ) using a 5-point scale according to combined assessments of alveolar congestion, hemorrhage, infiltration in the airspace or vessel wall, and thickness of alveolar wall/hyaline membrane formation damage. Each high-power field was recorded on a scale from 0 to 4 $(0=$ normal,$\quad 1=$ mild damage, $2=$ moderate damage, $3=$ severe damage, and $4=$ most severe damage) as described previously. ${ }^{16}$

\section{Electron Transmission Microscopy}

The electron transmission microscopy was estimated according to the method described. ${ }^{17}$ In brief, the right lungs of mice were washed twice with PBS, and fixed for $1 \mathrm{~h}$ in a solution containing $3.0 \%$ glutaraldehyde in $0.1 \mathrm{M}$ cacodylate buffer ( $\mathrm{pH}$ 7.2). After fixation, the samples were washed twice in the same buffer and then post-fixed in a solution containing $1 \%$ osmium tetroxide, $2 \mathrm{mM} \mathrm{CaCl}$, and $0.8 \%$ potassium ferricyanide in $0.1 \mathrm{M}$ cacodylate buffer (PH 7.2), dehydrated in acetone and embedded in Epon resin. Polymerization was performed at $60^{\circ} \mathrm{C}$ for 2 days. Ultra-thin sections were collected on 300-mesh copper grids, counter-stained with uranyl acetate and lead citrate, and examined using a Hitachi H-600 transmission electron microscope (Hitachi, Tokyo, Japan).

\section{Analysis of Pulmonary Microvascular Permeability and Pulmonary Edema}

Pulmonary vascular permeability was estimated by using the ratios of bronchoalveolar lavage fluid (BALF) to plasma 
b
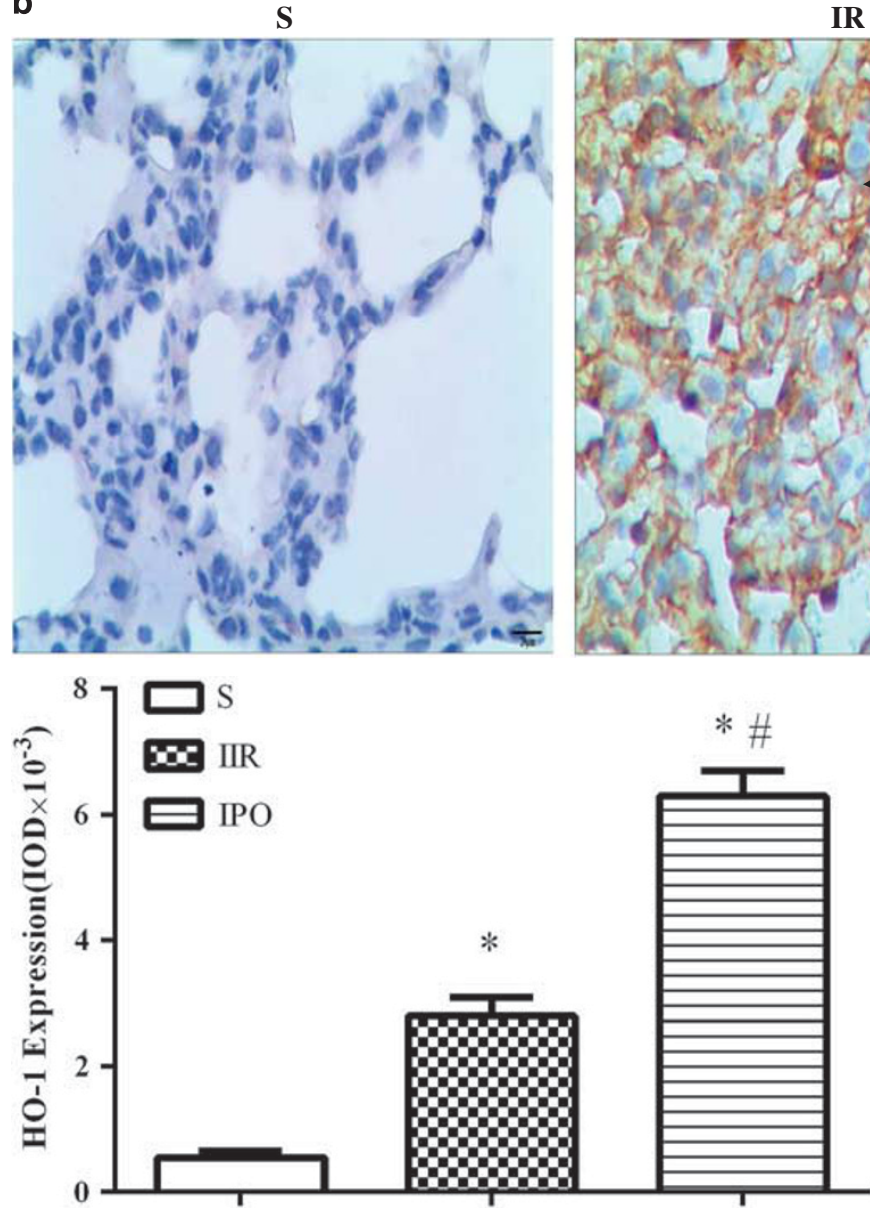

IR

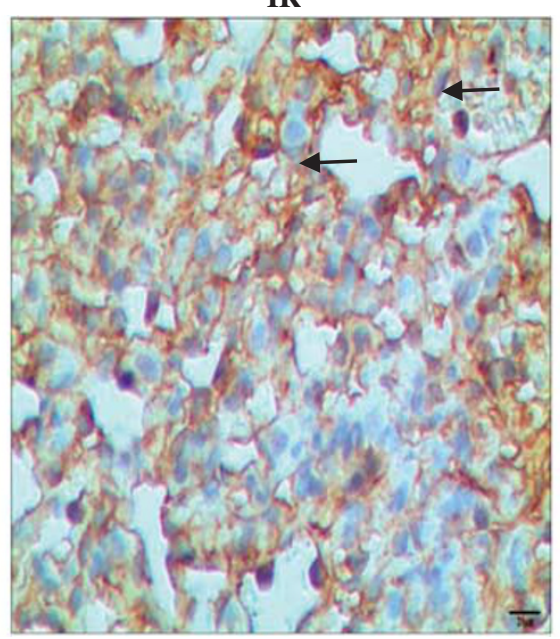

IPO

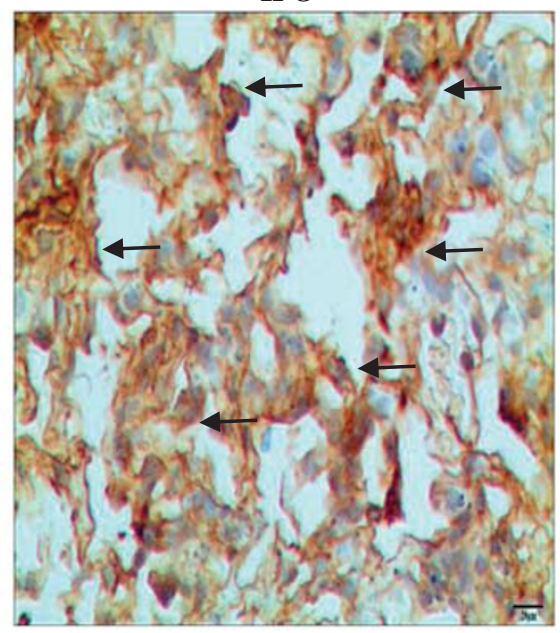

Figure 2 Continued.

protein (PP).$^{18}$ In brief, the left lungs were lavaged with $2 \mathrm{ml}$ PBS. A microvascular clamp was used to occlude the right hilum before lavage. BALF was immediately centrifuged $12000 \mathrm{~g}$ at $4{ }^{\circ} \mathrm{C}$ for $10 \mathrm{~min}$, and the supernatant was stored at $80^{\circ} \mathrm{C}$ until additional analysis. Total protein of BALF was measured using a standard commercial kit Sigma Chemical (St. Louis, MO, USA).

Pulmonary edema was detected by the mean wet/dry weight ratio of lung tissues. At the end of experiments, the lower lobe of the left lungs was removed from the thoracic cavity, weighed, and then placed in a drying oven at $80^{\circ} \mathrm{C}$ for $24 \mathrm{~h}$. After this drying procedure the specimens were reweighed, and the ratio of the weight before and after drying was calculated. Pulmonary edema was represented by an increase of this ratio.

\section{Immunohistochemistry Analysis}

Paraffin-embedded lung sections were stained using the Strept Avidin-Biotin Complex immunohistochemistry technique for HO-1 and Nrf2 detection. ${ }^{19}$ Brown staining in the cytoplasm and the nucleus were considered as the indicator of positive expression. Using the Image-Pro Plus version 6.0, results were evaluated semiquantitatively according to optical density values of positive expression.

\section{Western Blotting Analysis}

Endochylema and cellular nuclear proteins were extracted from frozen lung tissues using a nuclear extract kit according to the manufacturer's instructions. ${ }^{20}$ An equal amount of protein was loaded onto $12 \%$ SDS-PAGE at $100 \mathrm{~V}$ for $3 \mathrm{~h}$. After electrophoresis, proteins were transferred onto PVDF membranes at $200 \mathrm{~mA}$ for $2 \mathrm{~h}$. The transferred membranes were incubated overnight at $4{ }^{\circ} \mathrm{C}$ with rabbit polyclonal antibodies HO-1 or Nrf2 (1:200 dilution) against mouse in TBS-T containing 5\% no-fat milk. After washing three times with TBST, the membranes were incubated with anti-rabbit IgG conjugated to HRP (1:2000 dilution) in the same TBS-T for $2 \mathrm{~h}$ at room temperature. The immunoreactive bands were visualized with enhanced chemiluminescence and captured on X-ray film. The control was blotted against monoclonal Lamin-B or $\beta$-actin antibody. Optical density of the bands was measured using BandScan imaging analysis system.

\section{TNF- $a$, IL-6, and IL-10 Levels in Blood Plasma and Lung Tissues}

The inflammatory cytokine levels of TNF- $\alpha$, IL- 6 , and IL-10 in the blood plasma and lung tissues were quantified by using ELISA kits specific for mouse cytokines. ${ }^{21}$ All the steps 


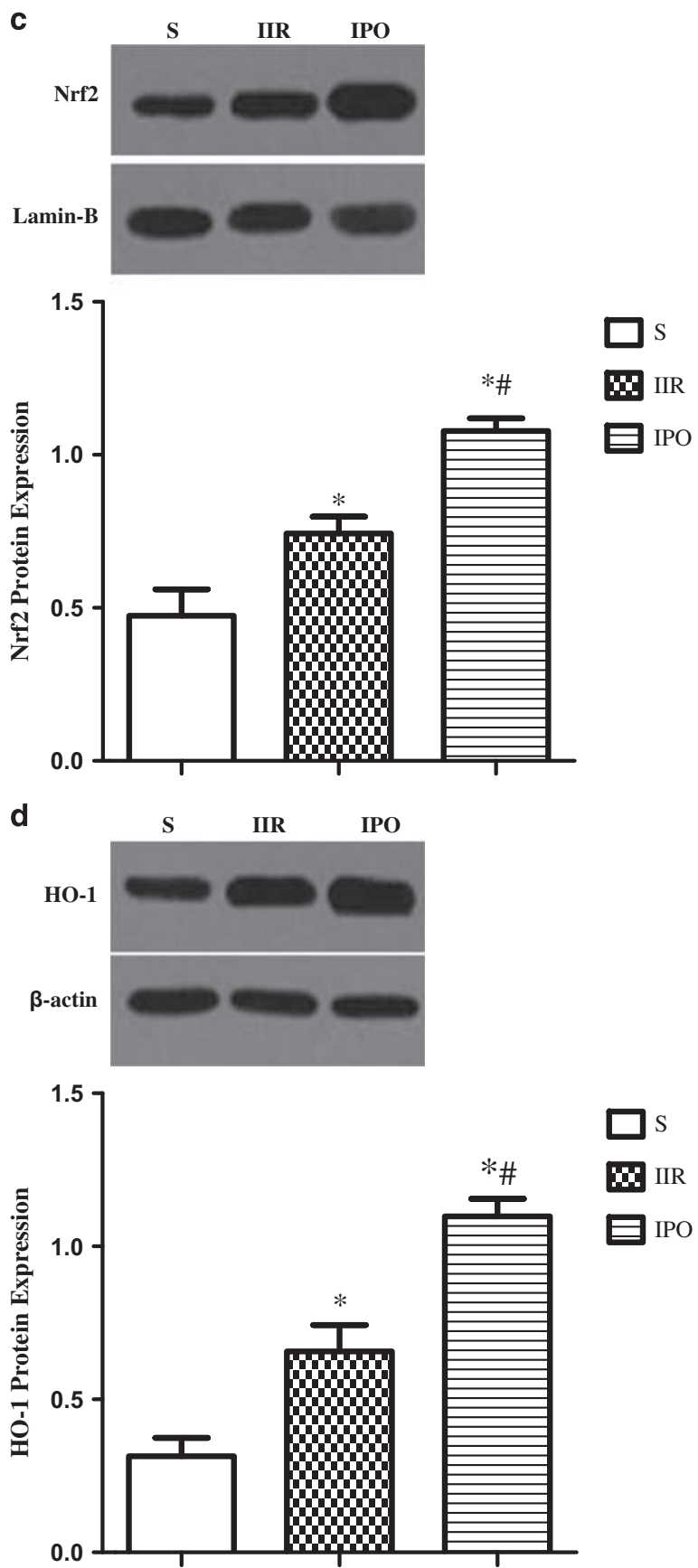

Figure 2 Continued.

involved were performed according to the manufacturer's instructions. Values were expressed as $\mathrm{pg} / \mathrm{ml}$.

\section{Measurement of Malondialdehyde (MDA) in Lung}

Tissues

After homogenizing on ice in normal saline, the homogenates were centrifuged at $12000 \mathrm{~g}$ for $15 \mathrm{~min}$ at $4^{\circ} \mathrm{C}$, and the MDA levels of the supernatants of the tissue samples were determined by using the thiobarbituric acid colorimetric method and MDA assay kit as previously described. ${ }^{22}$ Briefly, after mixing trichloroacetic acid with the homogenate and centrifugation, the super- natant was taken, and absorbance was measured using a spectrophotometer to monitor the reaction with TBA, and results were expressed as nanomole per milligram protein ( $\mathrm{nmol} / \mathrm{mg}$ protein).

Activity of Superoxide Dismutase (SOD) in Lung Tissues The SOD activity was estimated according to the method described. $^{23}$ In brief, pinephrine undergoes autoxidation rapidly at $\mathrm{pH} 10.0$ to produce adrenochrome, a pink colored product that was detected at $480 \mathrm{~nm}$ in kinetic mode using a UV-vis spectrophotometer. The amount of enzyme required 
a
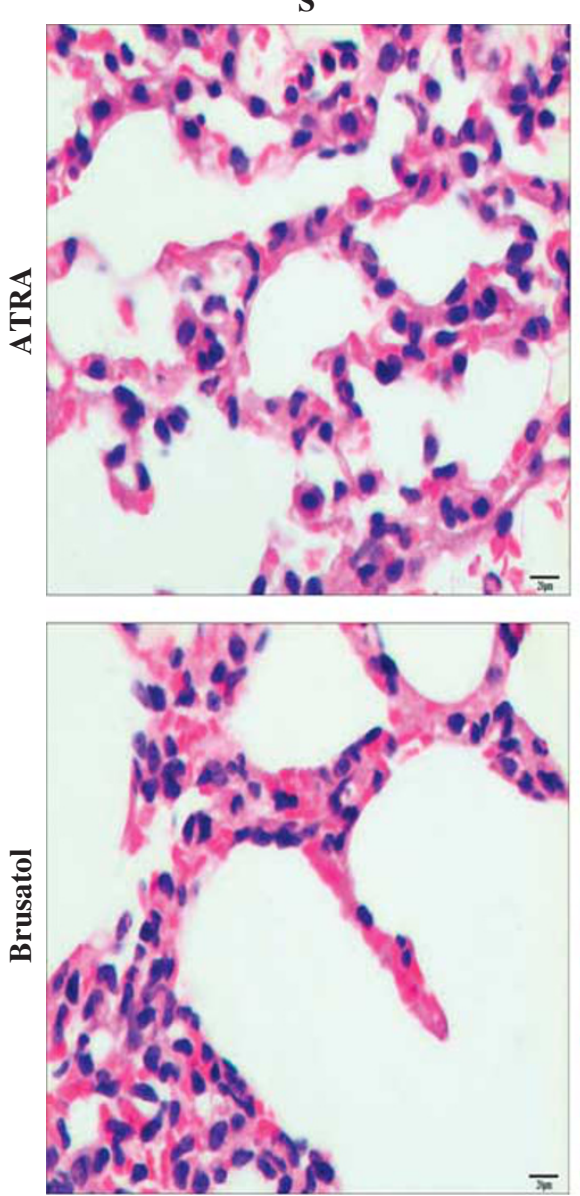

IR
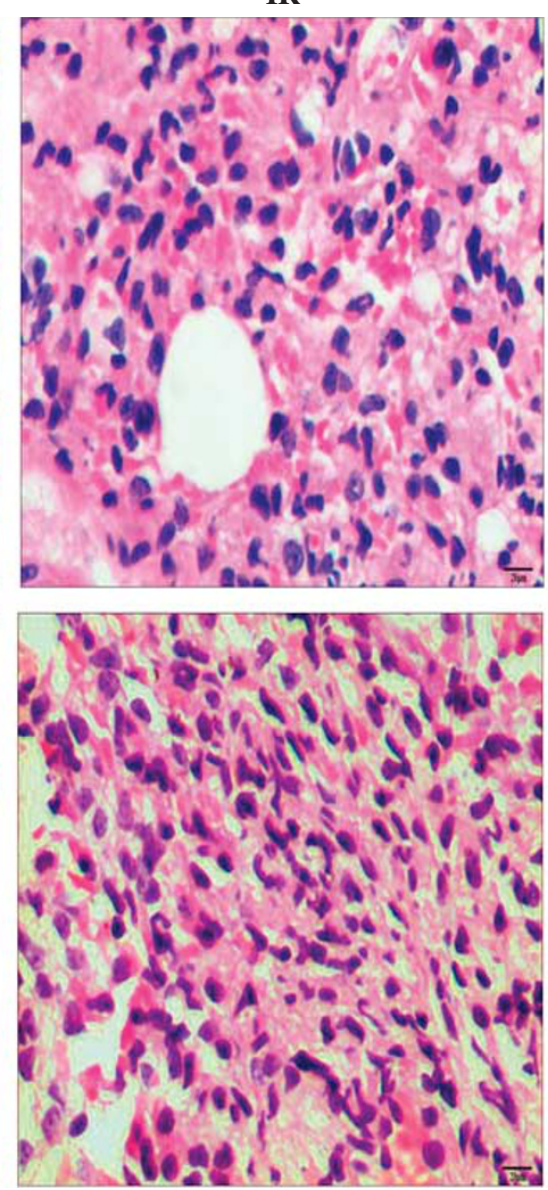

IPO
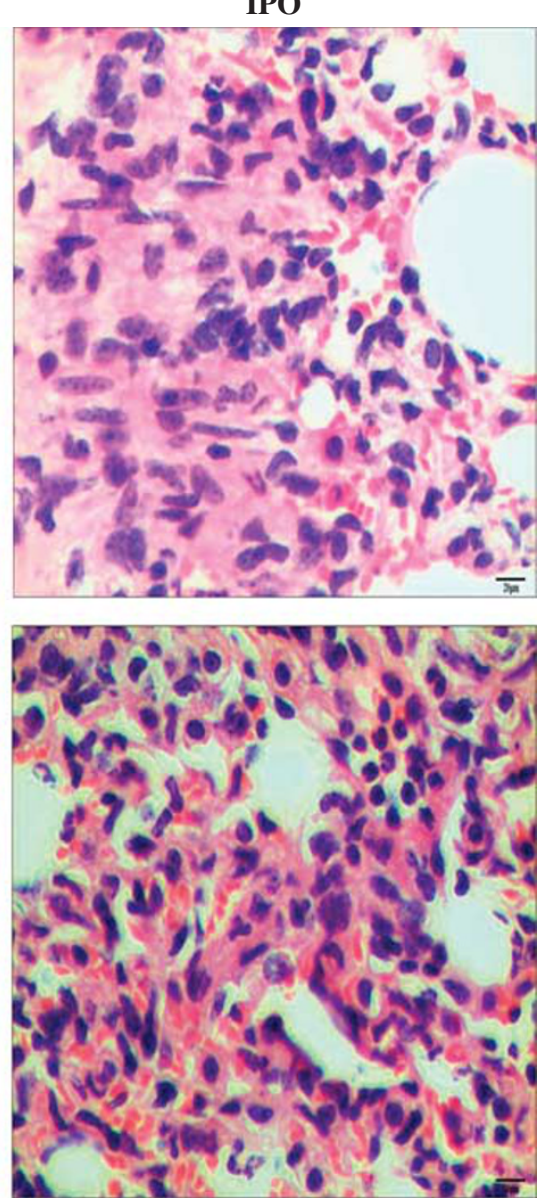

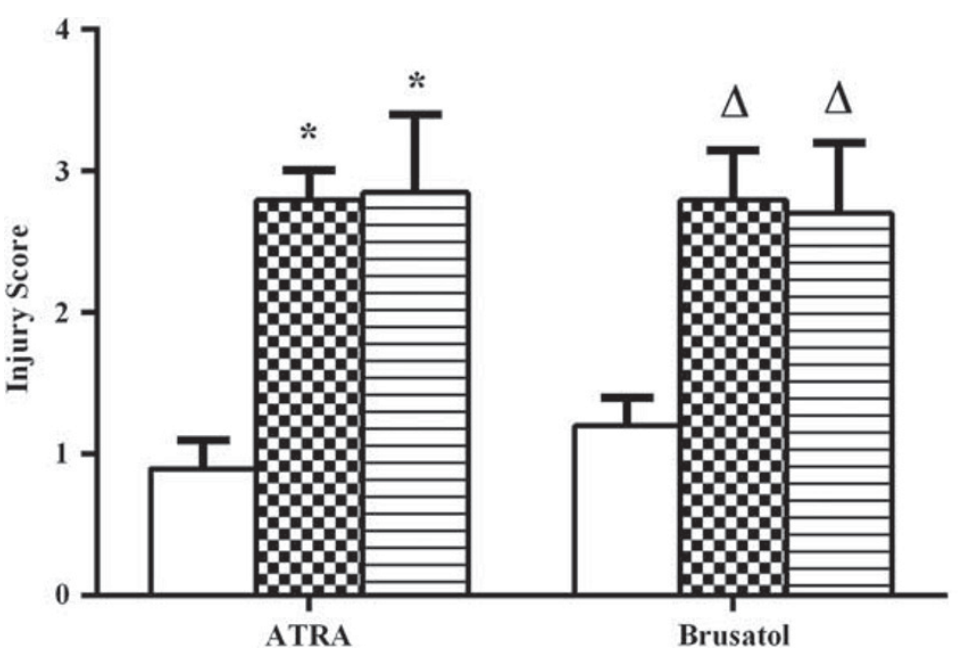

Antagonist $+\mathrm{S}$

Extagonist+IIR

E Antagonist+IPO

Figure 3 The protective effect of IPo against IIR-induced lung injury can be alleviated by Nrf2 antagonist. (a) The histopathologic changes of mice lung under a light microscope (H\&E staining). The injury changed deucedly in the IIR group after using ATRA and Brusatol, and there was no significantly amelioration for IPo after treated with ATRA (ATRA+IPo) or Brusatol (Brusatol+IPo). Scale bar, $20 \mu \mathrm{m}$. (b) The pulmonary electron microscopic evaluation ( $\times 8000)$. The injury changed deucedly in the IIR group after using ATRA, and there was no significantly amelioration after treated with IPo and ATRA (ATRA+IPO). Scale bar, $2000 \mathrm{~nm}$. Arrows indicate. (c, d) The change of the pulmonary microvascular permeability (c) and lung tissue wet/dry weight ratios (d) in lung tissue of mouse. The ratios of BALF to PP and the mean wet/dry weight ratio of lung tissues significantly increased in the IIR group after using ATRA and Brusatol, and they cannot be reversed by IPo treatment after using ATRA (ATRA+IPo) or Brusatol (Brusatol+IPo). Data presented as mean \pm s.d., $n=6-10$. ${ }^{*} P<0.05$ vs ATRA+S group, ${ }^{\triangle} P<0.05$ vs Brusatol+S group, one-way ANOVA and Tukey's post hoc test. 
b

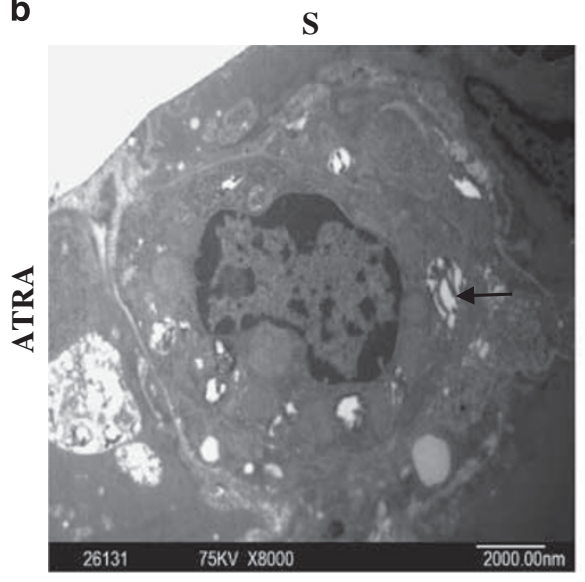

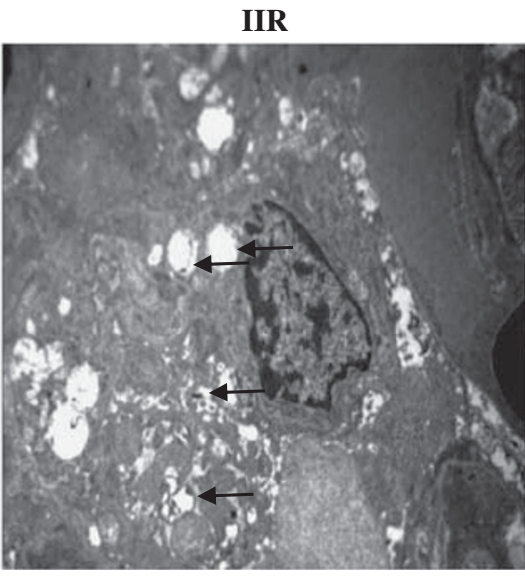

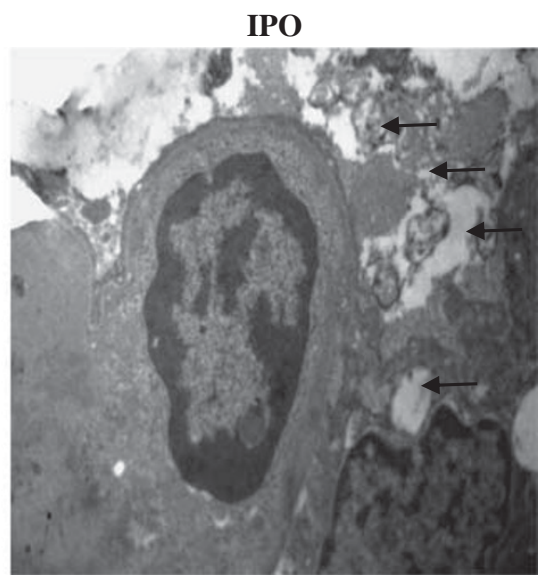

C

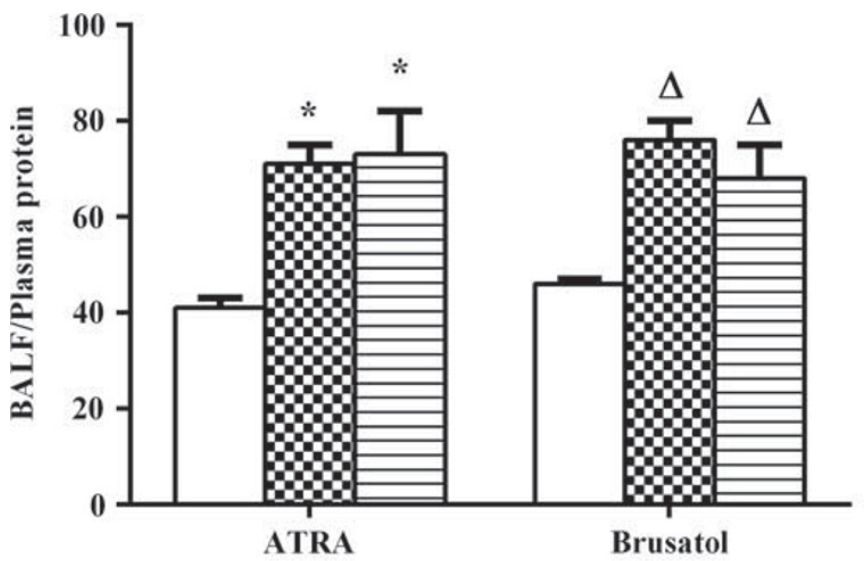

d

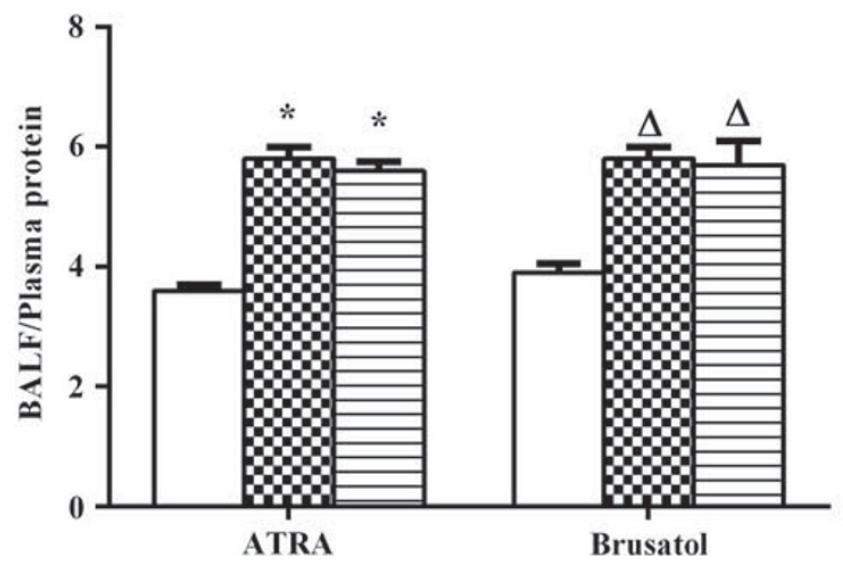

$\square$ Antagonist + S

E00 Antagonist+IIR

E Antagonist+IPO

$\square$ Antagonist + S

QD时 Antagonist+IIR

E Antagonist+IPO

Figure 3 Continued.

to produce $50 \%$ inhibition was defined as one unit of enzyme activity. The SOD activity was expressed as units/mg protein.

\section{Measurement of Glutathione Peroxidase (GSH-Px) Activity in Lung Tissue}

Activity of GSH-Px was determined by the velocity method using a GSH-Px kit. The reaction is initiated by the addition of $\mathrm{H}_{2} \mathrm{O}_{2}$. A series of enzymatic reactions are activated by GSH-Px in the homogenate that subsequently leads to the conversion of GSH (reduced glutathione) to GSSG (oxidized glutathione). The change in absorbance during the conversion of GSH to GSSG can be recorded spectrophotometrically at $412 \mathrm{~nm}$. The results are presented as nanomole per milligram protein (nmol/mg protein).

\section{Statistics Management}

The data are expressed as mean \pm s.d. The GraphPad Prism 5.0 statistical software program (GraphPad Software, San Diego, 
a
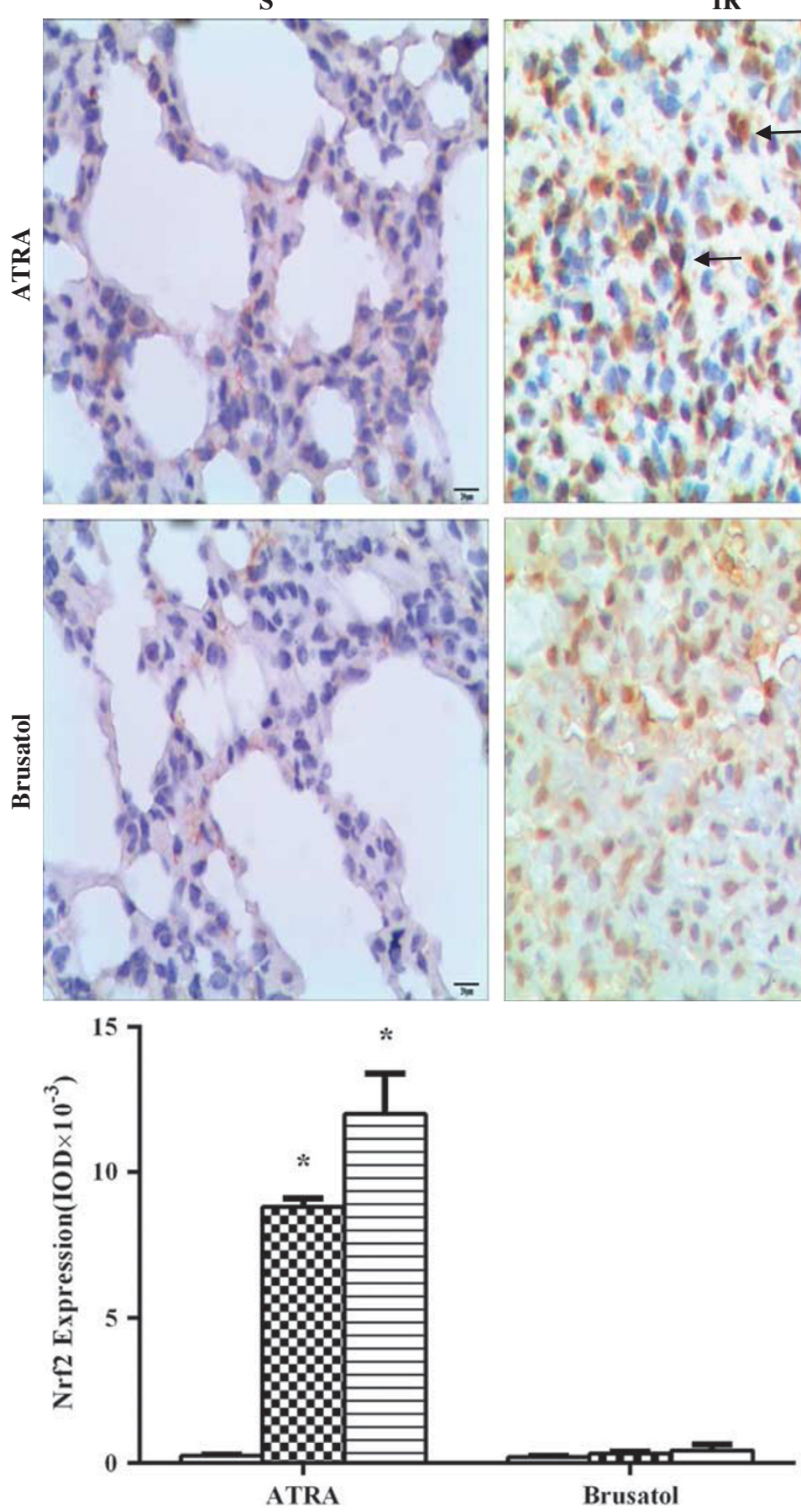

IR
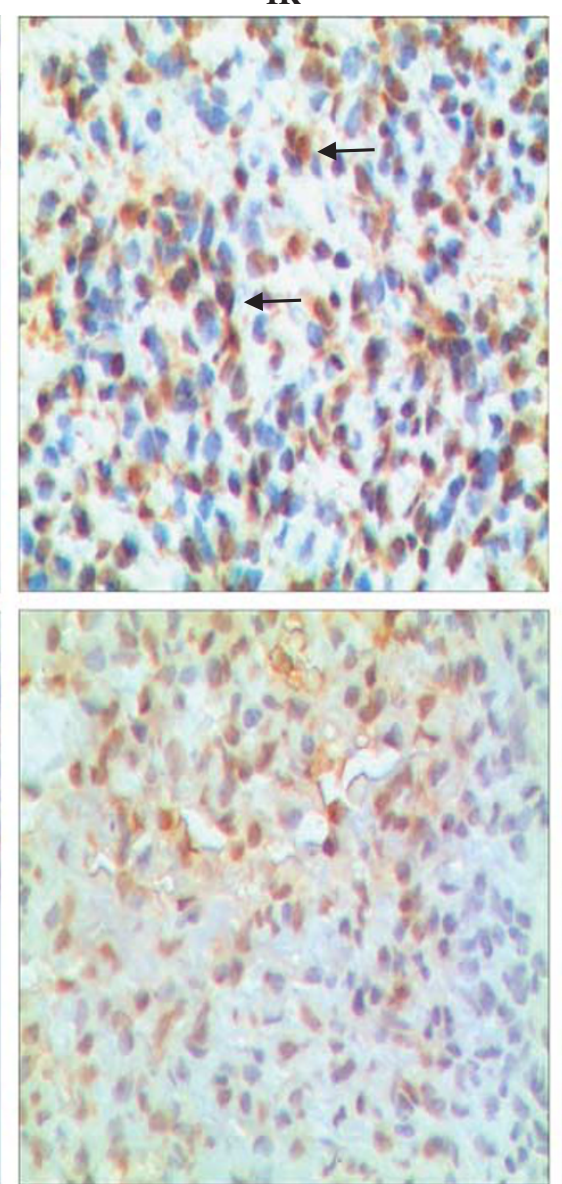

IPO
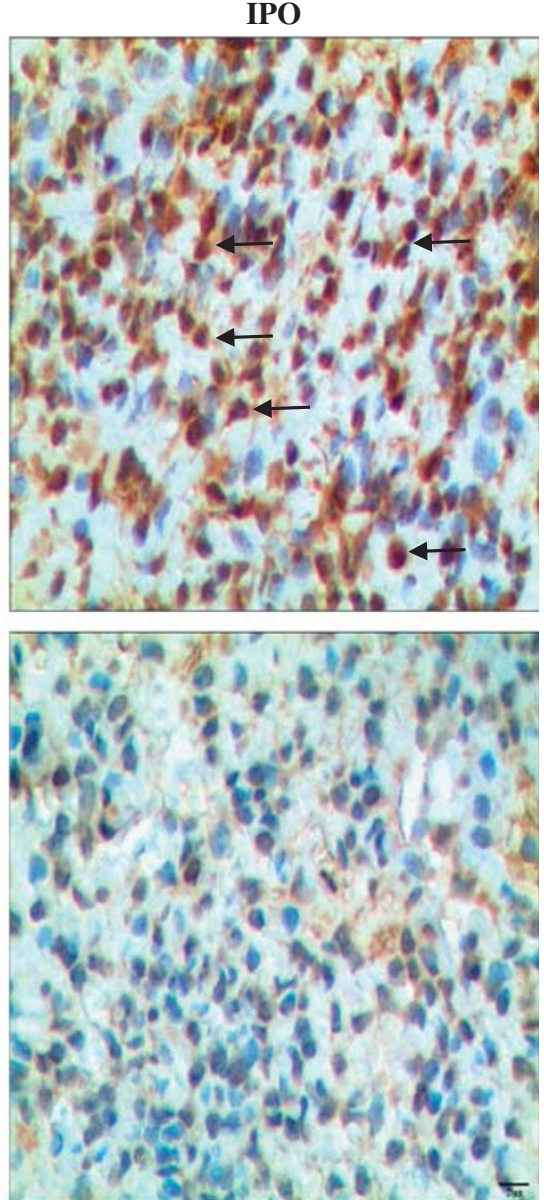

Antagonist $+S$

Extagonist+IIR

Antagonist+IPO

Figure 4 The expressions of Nrf2 and HO-1 in the lung. Lung injury after using Nrf2 antagonist. (a, b) A large number of lung tissue cells cytoplasm and nucleus showed brownish yellow or dark brown in the IIR group, and still a large number of lung tissue cells cytoplasm and nucleus showed brownish yellow or dark brown after treated with IPo. Scale bar, $20 \mu \mathrm{m}$. Arrows indicate (c, d). The expressions of Nrf2 and HO- 1 in the lung were detected by western blotting in each group. Lamin-B detected as a loading control of Nrf2 (c) by western blotting, and $\beta$-action detected as a loading control of HO-1 (d) by western blotting. Data presented as mean \pm s.d., $n=6-10 .{ }^{*} P<0.05$, vs ATRA+S group, one-way ANOVA and Tukey's post hoc test.

CA, USA) was used to set up our database and to calculate the results. Statistical evaluation of the data was performed by oneway analysis of variance (ANOVA), followed by Tukey's post hoc test. $P<0.05$ was considered as statistically significant.

\section{RESULTS}

\section{IPo Attenuates Intestinal Reperfusion-Induced ALI}

In order to investigate the underlying protective mechanisms of IPo, we studied the ALI induced by intestinal reperfusion 
b
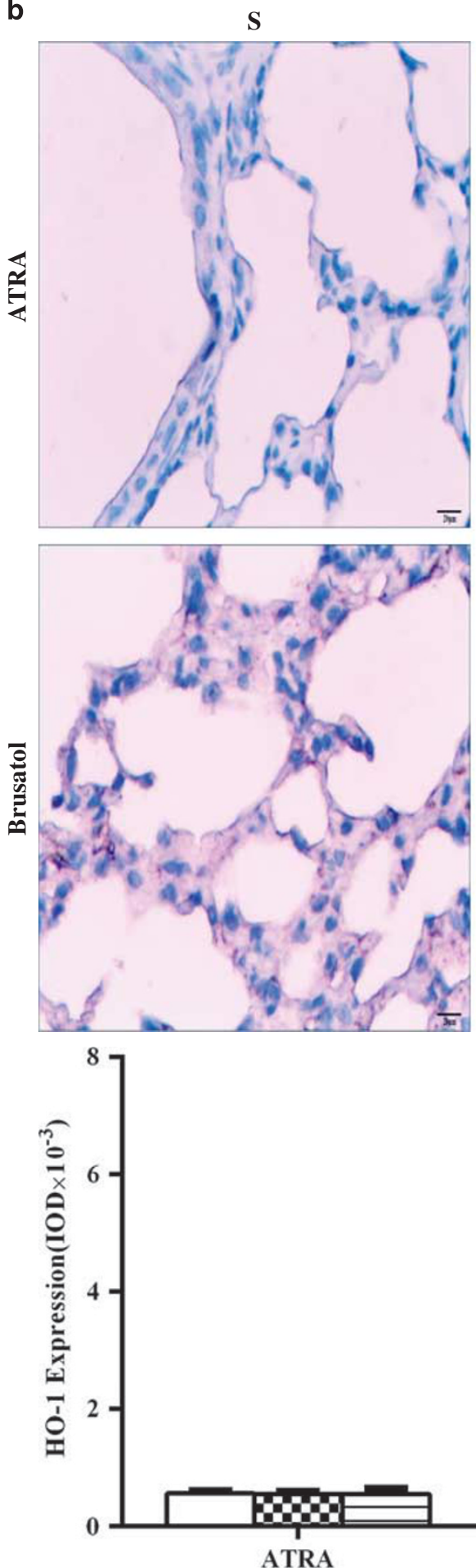

ATRA
IR
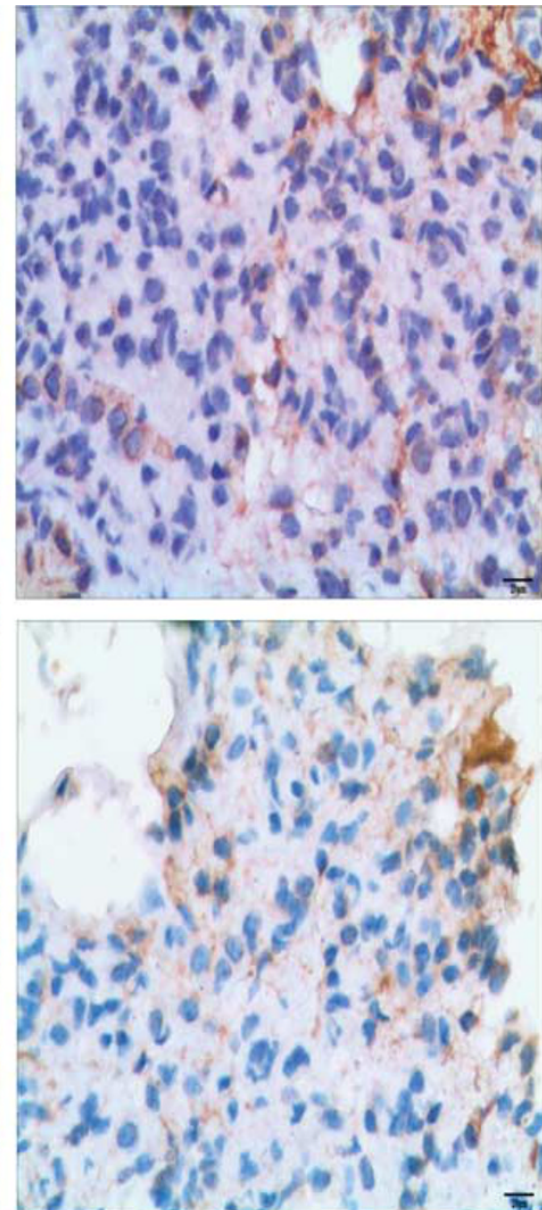
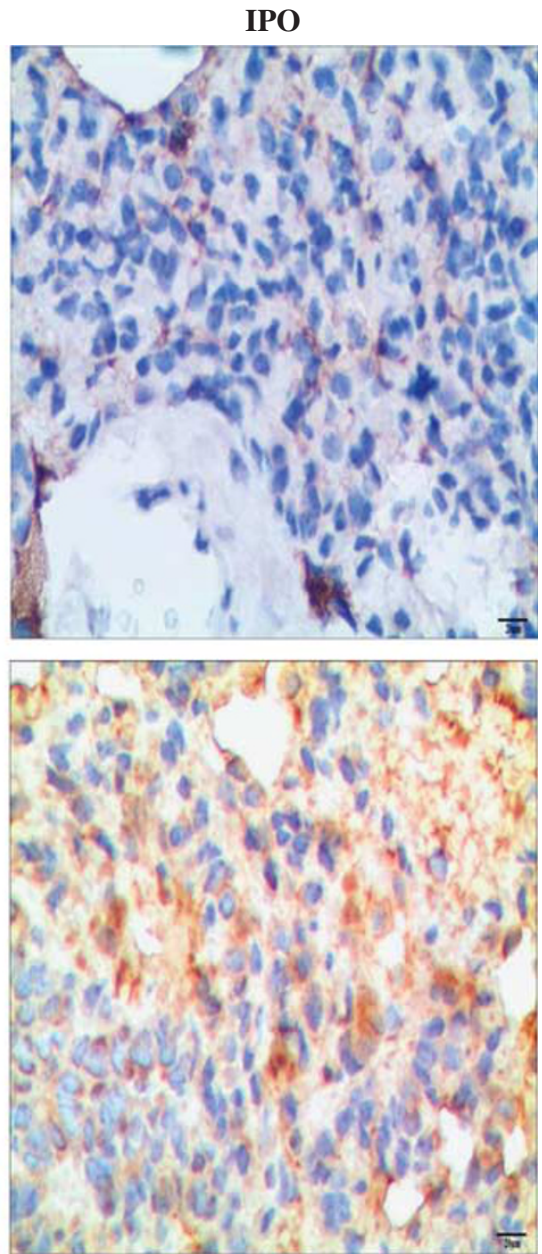

象

Antagonist $+\mathrm{S}$

00 Antagonist+IIR

Antagonist+IPO

Figure 4 Continued.

in the presence and absence of IPo. H\&E staining showed that IIR caused alveolar interstitial edema, hemorrhage, thickening of the alveolar wall, telangiectasis, and infiltration of inflammatory cells into the interstitial and alveolar spaces. These changes were dramatically diminished after
IPo (Figure 1a). Consistent with these results, transmission electron microscopy showed that the basement membranes of alveolar epithelium and capillary endothelium became swollen, widened, and with reduced or disappeared electronic density after 120 min reperfusion challenge. The 

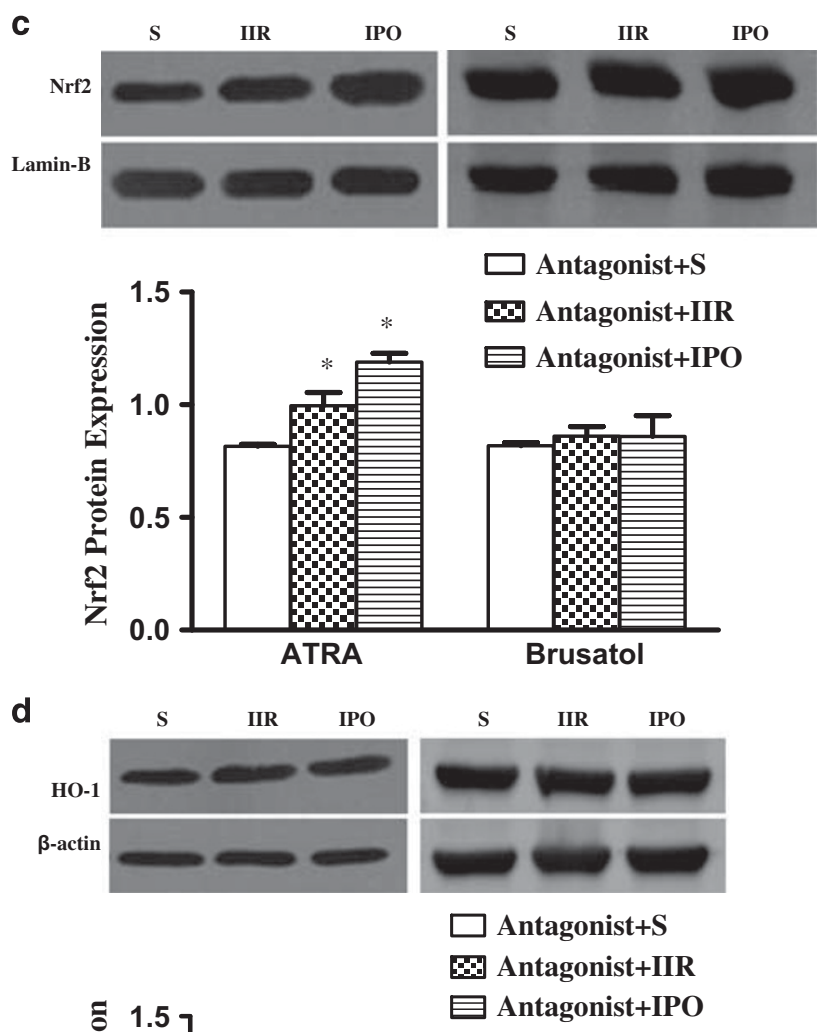

Figure 4 Continued.

mitochondria in type II alveolar cells became swollen, osmiophilic lamellar bodies turned emptied, and cellar ridge lodged or disappeared. Moreover, the conjunctions between the alveolar epithelial cells and the capillary endothelial cells were injured with gaps. There were marked attenuation of these pathologic alterations after mice were treated with IPo (Figure 1b).

Next, we examined the pulmonary microvascular permeability and pulmonary edema formation by the ratios of BALF to $\mathrm{PP}$ and the W/D lung weight ratios (Figures 1d and e, respectively). The ratios of BALF to PP in the IIR group were significantly higher than those in the $S$ group $(P<0.05$; Figure 1d). Treatment with IPo significantly reduced the pulmonary microvascular dysfunction induced by IIR $(P<0.05$; Figure $1 \mathrm{~d})$. Similarly, the wet/dry weight ratio of lung tissues in the IIR group was also significantly higher than that in the $S$ group $(P<0.05$; Figure $1 \mathrm{e})$. IPo treatment significantly decreased the IIR-related pulmonary edema $(P<0.05$; Figure 1e $)$.

\section{The Expression and Function of Nrf2 are Crucial in the Protection of IPO}

To determine the effect of Nrf2 on the protection of IPo, we first examined the expression of Nrf2 and HO-1 in the mouse lung tissue. We observed that Nrf2 $(P<0.05$; Figures 2a and c) and HO- $1(P<0.05$; Figures $2 \mathrm{~b}$ and $\mathrm{d})$ highly expressed in the group IIR, and further increased in the group IPO.

Then, we tested the inhibition potential of ATRA and Brusatol. ATRA cannot block the nuclear accumulation of Nrf2 but can reduce the binding of Nrf2 to the ARE, and then reduce the protein level of HO-1. Brusatol can selectively reduce the protein level of Nrf2 through enhanced ubiquitination and degradation of Nrf2. The degrees of lung injury did not obviously change in group IIR after the administration of Nrf2 antagonists, ATRA and Brusatol (Figure 3a). However, the protective effects of IPo were abolished by Nrf2 antagonists (Figures $3 \mathrm{a}$ and $\mathrm{b}$ ).

Meanwhile, we assessed the pulmonary microvascular permeability and pulmonary edema formation. There was 
a

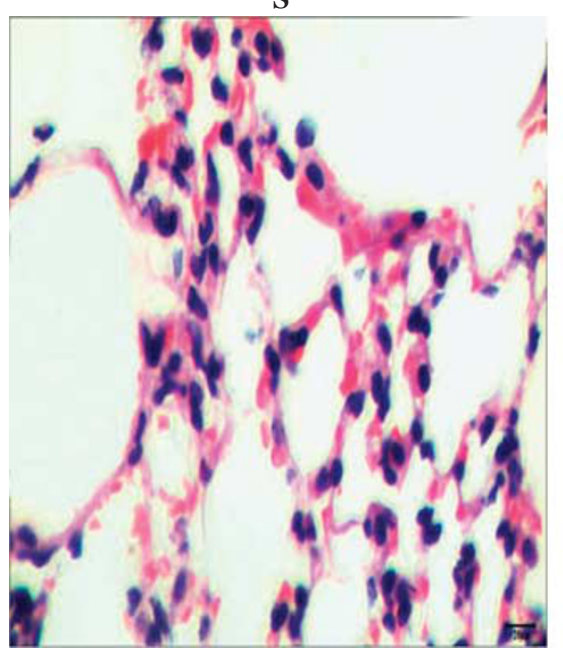

IR

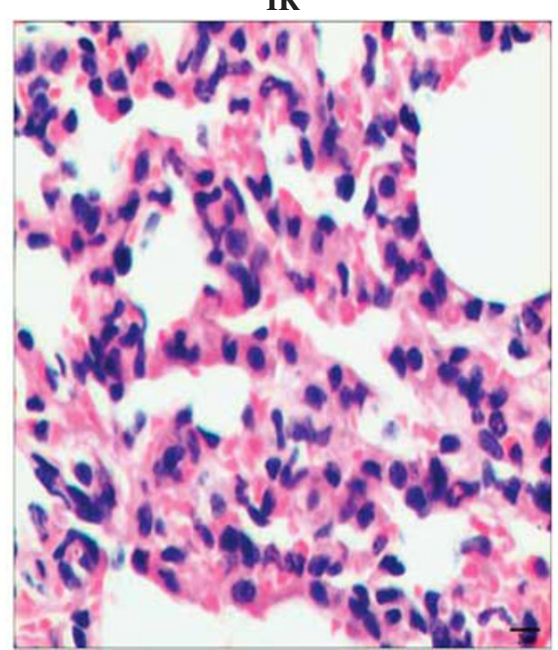

IPO

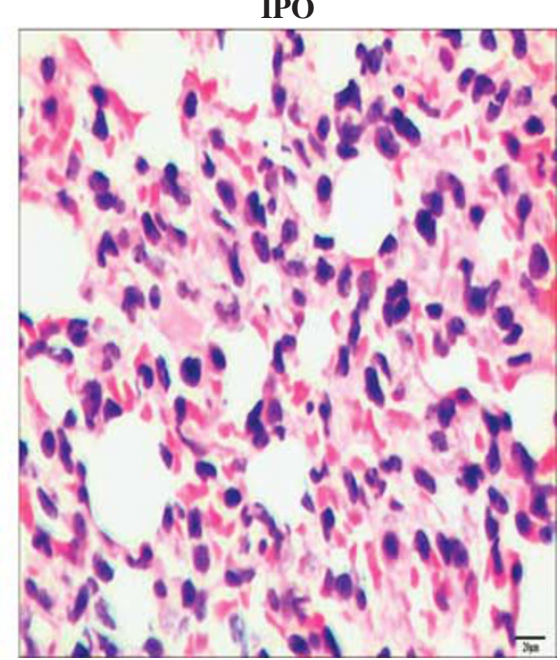

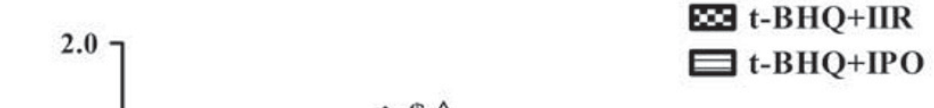
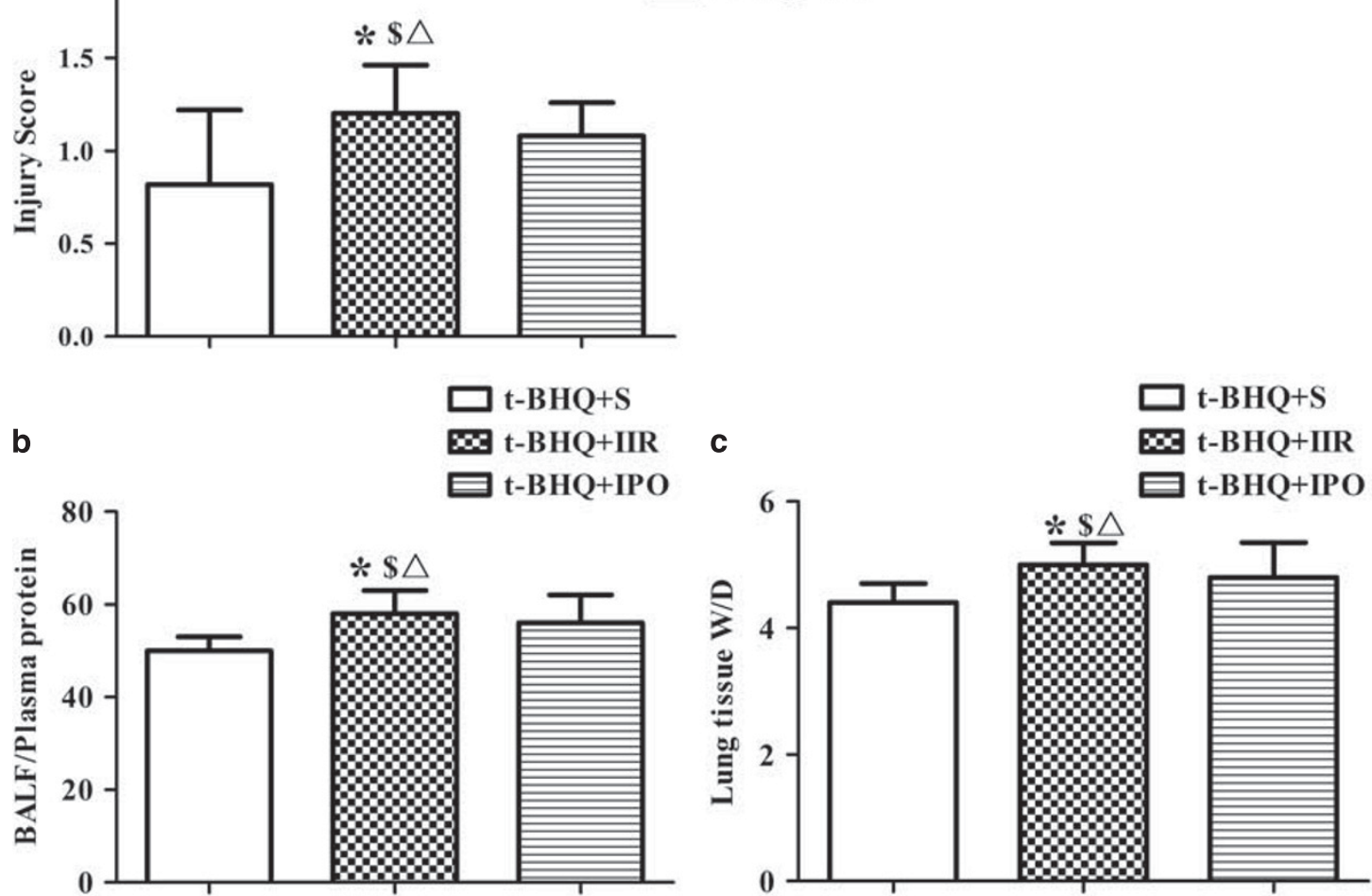

Figure 5 Nrf2 activation is involved in IPo-induced protection against lung injury induced by IIR. (a) The histopathologic changes of mice lung after using t-BHQ under a light microscope ( $\times 400, \mathrm{H} \& \mathrm{E}$ staining). The lung injury induced by IIR was markedly alleviated changed deucedly after using $\mathrm{t}-\mathrm{BHQ}$, and there was no significant difference in the $\mathrm{S}$, IIR, and IPo groups after using t-BHQ. Scale bar, $20 \mu \mathrm{m}$. (b.c) The change of the pulmonary microvascular permeability (b) and lung tissue wet/dry weight ratios (c) in lung tissue of mouse. There was no significant difference in the $S$, IIR, and IPo groups after using t-BHQ. (d, e) The expressions of Nrf2 (d) and HO-1(e) were detected by immunohistochemistry with the Streptomyces avidin-peroxidase connection (SP) method. (f, $\mathbf{g})$ The expressions of Nrf2 and HO-1 in the lung were detected by western blotting in each group. Lamin-B detected as a loading control of Nrf2 (f) by western blotting, and $\beta$-action detected as a loading control of HO-1 (g) by western blotting. Data presented as mean \pm s.d., $n=6-10$. ${ }^{*} P<0.05$, vs IIR group, ${ }^{\$} P<0.05$, vs ATRA+S group, ${ }^{\Delta} P<0.05$, vs Brusatol $+S$ group, ${ }^{\circ} P<0.05$, vs $\mathrm{t}-\mathrm{BHQ}+\mathrm{S}$ group, one-way ANOVA and Tukey's post hoc test. 


\section{d}
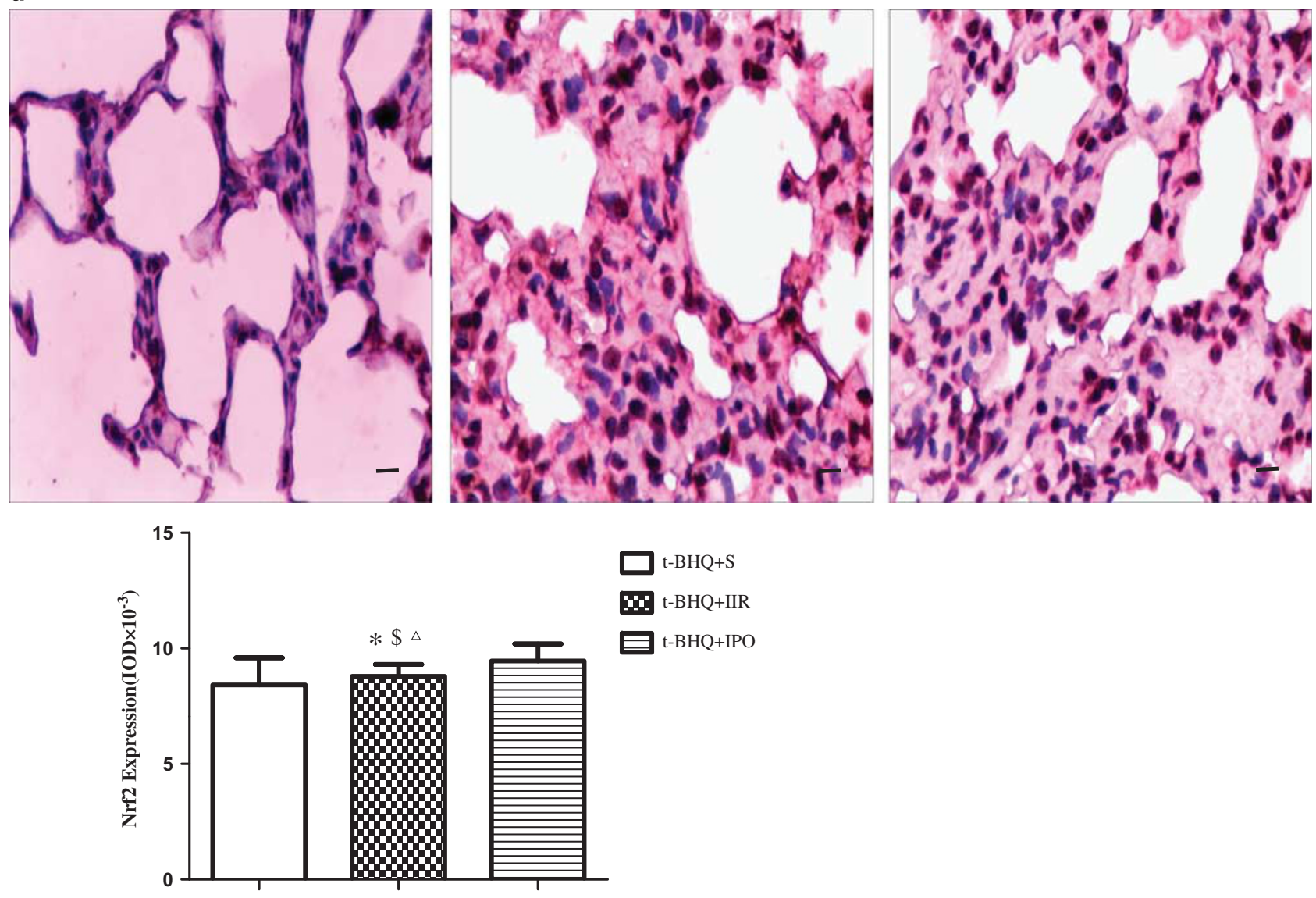

Figure 5 Continued.

no significant difference in the pulmonary microvascular permeability between the IIR group and the IPo group after Nrf2 antagonist treatment (Figure 3c). Similarly, there were no differences in pulmonary edema between IIR and IPo groups following antagonism of Nrf2 (Figure 3d). Moreover, morphometric analysis was performed on lung tissue, and we observed that Nrf2 increased in group IIR and highly expressed in group IPO $(P<0.05$; Figure 4a) after using ATRA, but it did not change in the groups IIR and IPo after using Brusatol (Figure 4a). Western blotting showed the same trend as immunohistochemistry analysis after using Nrf2 antagonist (Figure 4c). Meanwhile, administration of Nrf2 antagonists precluded an increase in expression of HO-1 in lung tissue as there was no significant difference in IPO group, $\mathrm{S}$ and IIR groups (Figures $4 \mathrm{~b}$ and $\mathrm{d}$ ).

Conversely, we tested the change of lung injury and Nrf2 expression after treated with an Nrf2 agonist, t-BHQ. The degree of lung injury by the IIR group was reversed by $\mathrm{t}-\mathrm{BHQ}$ treatment (Figure 5a). Moreover, t-BHQ treatment prevented the pulmonary microvascular permeability and pulmonary edema deficits induced by IIR (Figures $5 \mathrm{~b}$ and $\mathrm{c}$ ). Consistent with these observations, Nrf2 and HO-1 expression in lung tissue, as measured by immunohistochemistry and western blotting, was significantly increased after treatment with $\mathrm{t}-\mathrm{BHQ}$ (Figures $5 \mathrm{~d}-\mathrm{g}$ ).

\section{Nrf2 Regulates the Expression of Inflammatory Cytokines in Plasma and Lung Tissues}

Next, we investigated the changes in expression of inflammatory cytokines in plasma and lung tissues. The levels of TNF- $\alpha$ and IL- 6 in plasma and lung tissues increased markedly in the IIR group $(P<0.05)$, which were significantly attenuated by IPo $(P<0.05$; Figures $6 \mathrm{a}, \mathrm{b}, \mathrm{d}$ and $\mathrm{e})$. The decreased levels of TNF- $\alpha$ and IL- 6 induced by IPO in the plasma and lung tissues were prevented with $\mathrm{Nrf2}$ antagonist treatment $(P<0.05$; Figures $6 \mathrm{a}, \mathrm{b}, \mathrm{d}$ and $\mathrm{e})$. The increased levels of TNF- $\alpha$ and IL- 6 induced by IIR were precluded after $\mathrm{t}-\mathrm{BHQ}$ treatment $(P<0.05$; Figures $6 \mathrm{a}, \mathrm{b}, \mathrm{d}$ and $\mathrm{e})$.

The expression of IL-10 in the IIR group decreased dramatically as compared with that in the $S$ group $(P<0.05$; Figures $6 \mathrm{c}$ and $\mathrm{f})$. IPo significantly reversed the decreased trend of IL-10 induced by IIR $(P<0.05)$, which was blocked by treatment with $\mathrm{Nrf} 2$ antagonists $(P<0.05$; Figures $6 \mathrm{c}$ and $\mathrm{f}$ ). The decreased trend of IL-10 induced by IIR was precluded following t-BHQ treatment $(P>0.05$; Figures $6 \mathrm{c}$ and $\mathrm{f}$ ). 

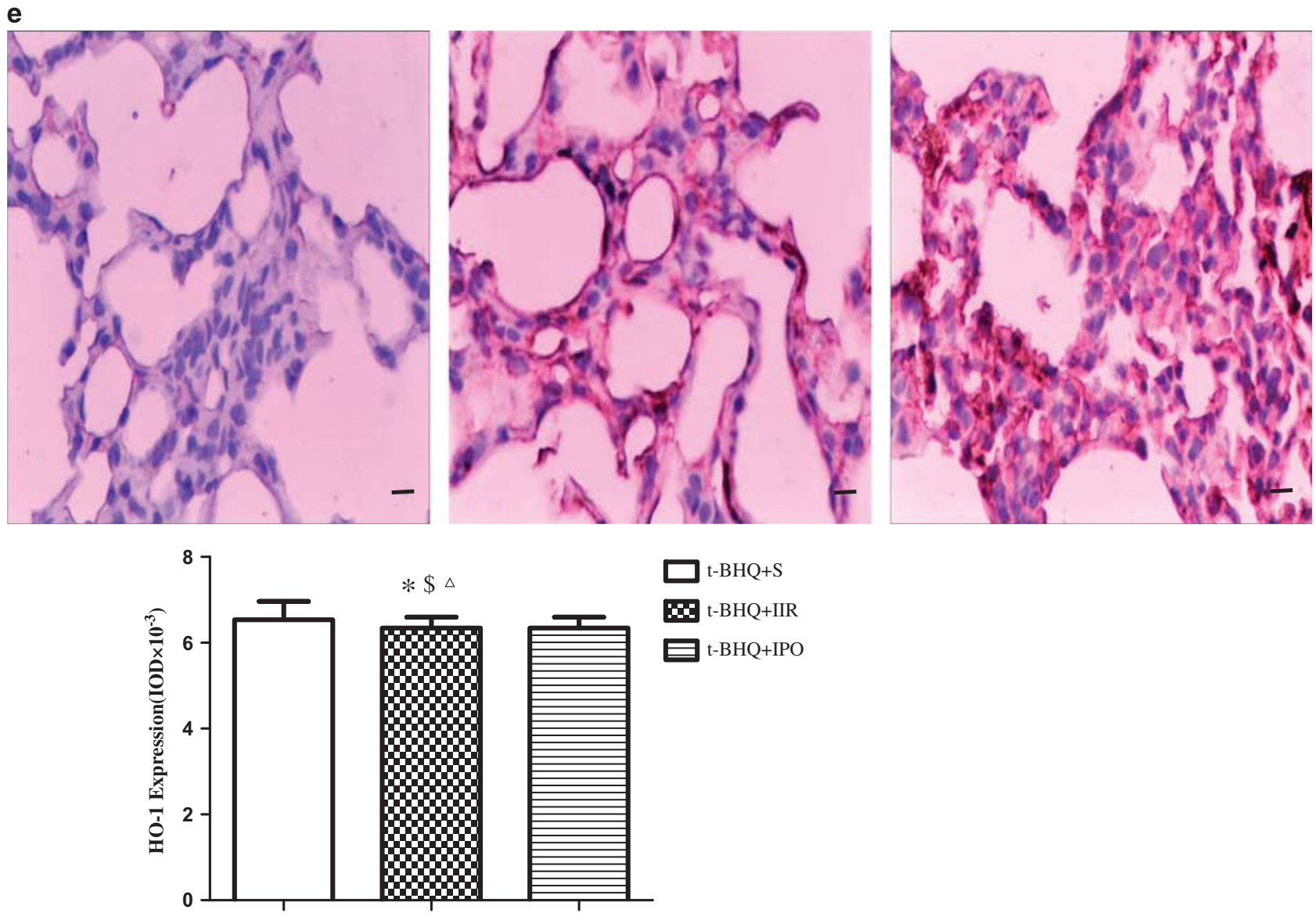

Figure 5 Continued.

\section{Nrf2 Regulates the MDA Expression, SOD Activity and GSH-Px Activity}

We next assessed the expression of MDA, activity of SOD and GSH-Px. We found that the levels of MDA in the IIR group were increased significantly compared with the $S$ group $(P<0.05$; Figure $7 \mathrm{a})$. Conversely, IPo significantly decreased the levels of MDA $(P<0.05$; Figure $7 \mathrm{a})$, and the decreased MDA levels in the IPo group were blocked after treatment with Nrf2 antagonist $(P<0.05)$. t-BHQ treatment significantly inhibited the increased MDA levels induced by IIR $(P<0.05$; Figure $7 \mathrm{a})$.

The activity of SOD and GSH-Px decreased dramatically in the IIR group as compared with the $S$ group $(P<0.05$; Figures $7 \mathrm{~b}$ and $\mathrm{c})$. IPo significantly increased the activity of SOD and GSH-Px compared with the IIR group $(P<0.05)$, and the increased activity of SOD and GSH-Px was blocked after treatment with $\mathrm{Nrf2}$ antagonists $(P<0.05)$. t-BHQ treatment significantly increased the activity of SOD and GSH-Px in the IIR group compared with the control IIR $\operatorname{group}(P<0.05$; Figures $7 \mathrm{~b}$ and $\mathrm{c})$.

\section{DISCUSSION}

We have demonstrated that IPo significantly attenuated lung histological damage, pulmonary microvascular permeability, pulmonary edema, and increased lung SOD and GSH-Px activity. IPo can attenuate lung injury induced by IIR and reduce the systemic inflammatory response in mice by upregulating the master transcriptional factor Nrf2 and increased activation of antioxidant defense enzymes HO-1 in the lung. The beneficial effects of IPo were abolished or severely attenuated by concomitant treatment with the Nrf2 antagonist. Our studies suggest that upregulation of Nrf2 expression plays a critical role in IPo-mediated protective effects against IIR-induced lung injury through attenuation of oxidative stress and systemic inflammatory response.

IPo is defined as rapid intermittent interruptions of blood flow in the early phase of reperfusion and mechanically alters the hydrodynamics of reperfusion. The beneficial effects of IPo against ischemia/reperfusion (IIR) injury have been demonstrated in multiple organs. ${ }^{6,24}$ Some studies have demonstrated the protection of IPo against ALI induced by IIR, ${ }^{7,25,26}$ and that IPo could attenuate systemic proinflammatory cytokine release and lung IIR injury by enhancing the expression of $\mathrm{HO}-1$, in response to various pathophysiologic stresses. However, the underlying mechanisms governing the increase of HO-1 protein expression or activity under the conditions of IIR and IPo intervention have not been explored. Nrf2 responds rapidly to oxidative stress, 

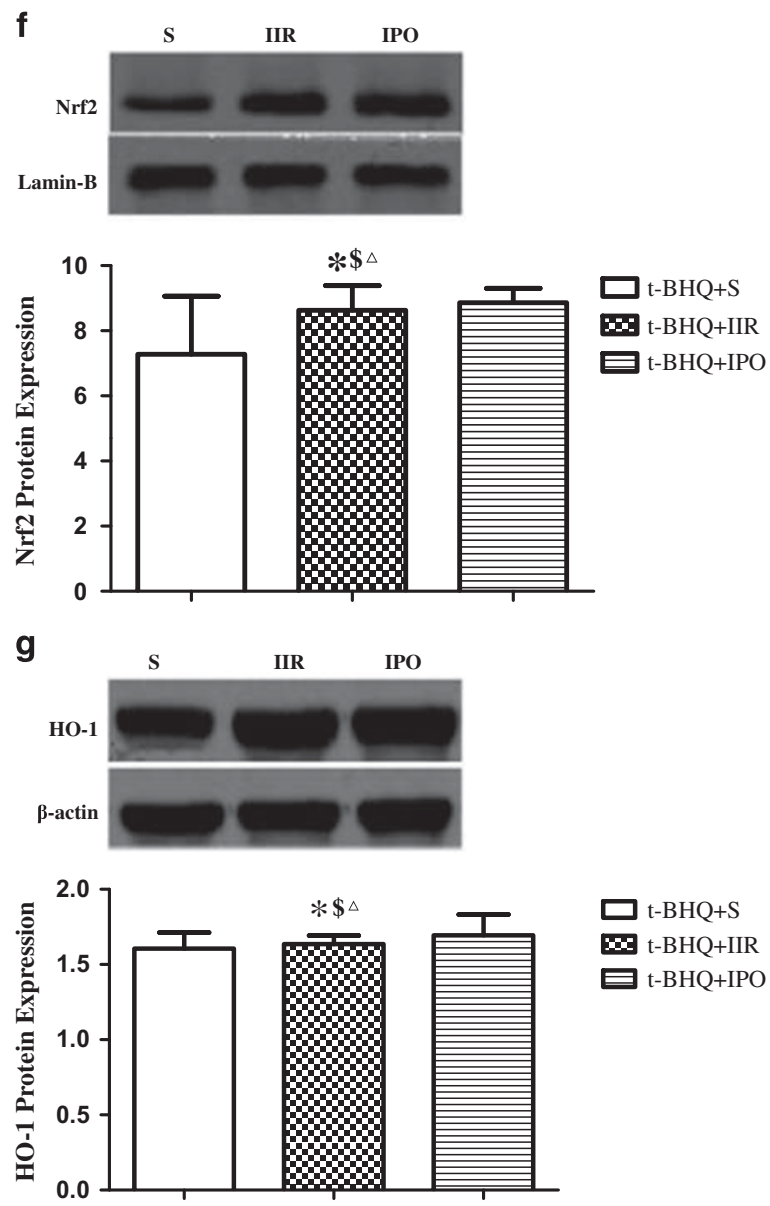

Figure 5 Continued.

an important pathogenic factor in most forms of ALI. ${ }^{27,28}$ In the present study, we found that IPo can attenuate IIR-induced lung injury, and that IPo treatment also significantly attenuated pulmonary microvascular permeability, pulmonary edema. Furthermore, IPo treatment upregulated the expression of $\mathrm{Nrf}_{2}$ and $\mathrm{HO}-1$ in lung. The results suggest that Nrf2 signal might be a vital mechanism to protect the organ from IIR-induced further damage.

As an important endogenous mechanism to mitigate oxidative stress, Nrf2 has been identified as a key regulator in mediating the induction of genes encoding several antioxidant enzymes via the ARE in response to stressful stimuli. ${ }^{9,10,29}$ Nrf2 activation and the subsequent cytoprotective gene induction not only promotes the restoration of balance between oxidants and antioxidants in response to stressful stimuli but also induces enhanced transcription of target antioxidant/detoxifying genes to enhance cytoprotection. Nrf2 confers a protective role against oxidative stress and xenobiotic stress, including hyperoxia, mechanical ventilation, and cigarette smoke in the lung. ${ }^{10} \mathrm{Nrf} 2$ is released from Keap-1 repression under stress conditions and translocates to the nucleus where it transcribes a number of antioxidant and/or detoxification genes, such as $\mathrm{HO}-1$ and $\mathrm{NAD}(\mathrm{P}) \mathrm{H}$ quinone oxidoreductase 1 (NQO-1). ${ }^{30}$ Recent studies ${ }^{10,29}$ also indicated that the protective effects of $\mathrm{Nrf} 2$ against lung injury induced by IIR occur possibly through transcriptional activation of antioxidant defense enzymes such as HO-1 in the lung. HO-1 activation is an indispensable event in a variety of lung diseases, including ALI/ARDS, asthma, and respiratory viral infections, systemic inflammatory response syndrome, occupational and environmental lung disease. The upregulation of pulmonary HO-1 expression is now considered as an important part of the host anti-injury effect on ALI. ${ }^{31}$ Our results showed that lung Nrf2 transcription factor and HO-1 increased dramatically after IIR challenge, which suggested that lung Nrf2 and HO-1 increased dramatically by the stimulation of IIR to resist the lung injury. Meanwhile, the results also showed increased IIR-induced lung injury, suggesting that the protective effects produced by increased expression of Nrf2 and HO-1 were still not sufficient to fully protect pulmonary tissue from IIR-induced injury. Strikingly, IIR-induced lung injury was reversed by IPo and significantly increased Nrf2 and HO-1 expression. The results indicate that increased $\mathrm{Nrf} 2$ and $\mathrm{HO}-1$ play an important role in the beneficial effect induced by IPo treatment. 

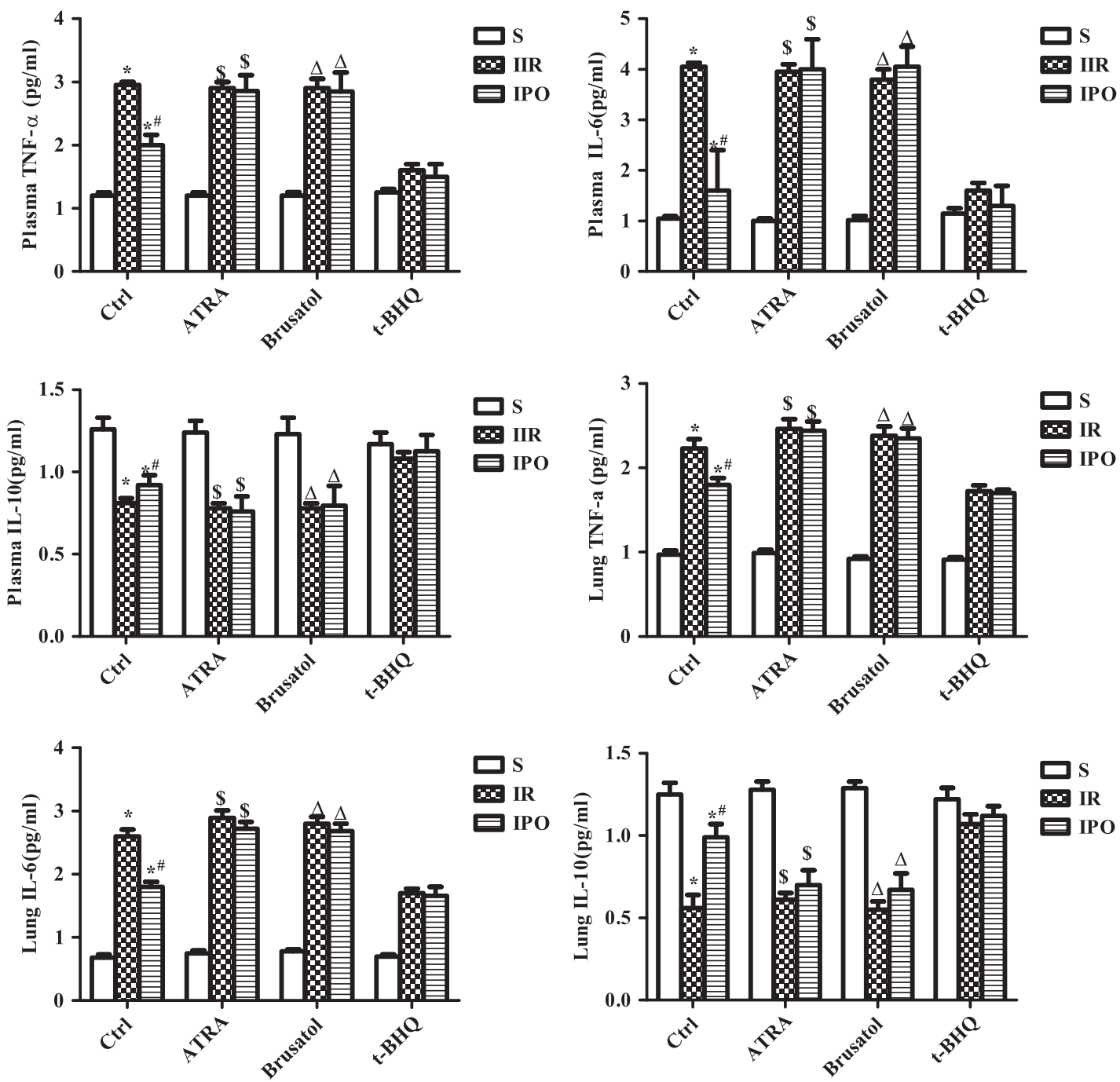

Figure 6 The plasma levels of inflammatory cytokines (a) TNF- $a$, (b) IL-6, and (C) IL-10 in each group. Data presented as mean $\pm s . d ., n=6-10$. ${ }^{*} P<0.05$, vs $S$ group, ${ }^{\#} P<0.05$, vs IIR group, ${ }^{\$} P<0.05$, vs ATRA+S group, ${ }^{\triangle} P<0.05$, vs Brusatol+S group, $P<0.05$, vs t-BHQ+S group, one-way ANOVA and Tukey's post hoc test.

Strikingly, the use of Nrf2 antagonists, ATRA and Brusatol, prevented the increase of $\mathrm{HO}-1$ mediated by IPo and precluded the beneficial effects of IPo in reducing oxidative stress and systemic inflammatory response. Furthermore, our data showed that treatment with $\mathrm{t}-\mathrm{BHQ}$, a Nrf2 agonist, has a similar protective effect as IPo. These results indicate that Nrf2 is crucial in the protective effect of IPo against IIR-induced lung injury, and this is a vital mechanism through which Nrf2 signaling is upregulated in IIR to protect the cell from further damage. This is confirmed by the findings that $\mathrm{Nrf} 2$ causes protective effects by regulating HO-1 production. ${ }^{10,29}$

IIR challenge results in translocation of bacteria and toxins, which amplifies systemic inflammation and oxidative stress, and leads to ALI and ARDS.,3,4,32 Oxidative stress is a disturbance in the balance between the production of free radicals and antioxidant defense systems. Free radicals are generated in the form of reactive oxygen species (ROS), including superoxide anions, hydrogen peroxide, hydroxyl radicals, and singlet oxygen. ${ }^{33}$ Oxidative stress also contributes to IIR-induced ALI not only through direct injurious effects including lipid peroxidation and apoptosis but also through involvement in the molecular mechanisms that control intestinal inflammation. ${ }^{34}$ Overproduction of ROS accompanied by a reduction in endogenous antioxidative defense causes destructive and irreversible oxidative damage to various cellular components, such as lipids, proteins, and DNA. Moreover, increased production of ROS increases oxidative stress, leading to cellular dysfunction and cell death during the I/R injury. ${ }^{35}$ The upregulation of transcription factor Nrf2 and ARE-mediated gene products, such as HO-1, maintains redox homeostasis and affects the inflammatory response. ${ }^{36}$ In this study, IPo significantly increased lung SOD and GSH-Px activity, and reduced 
a

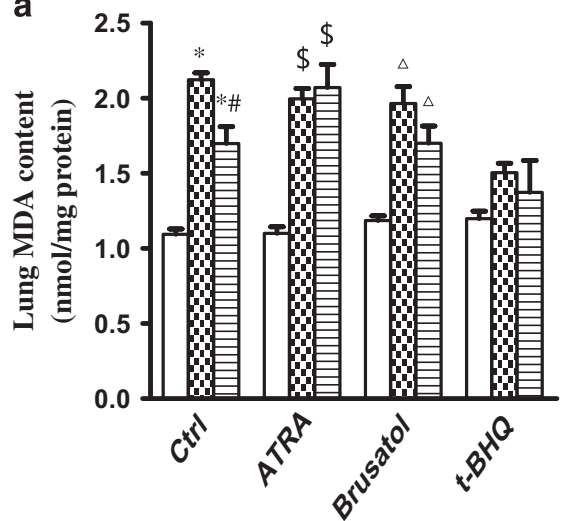

\section{b}
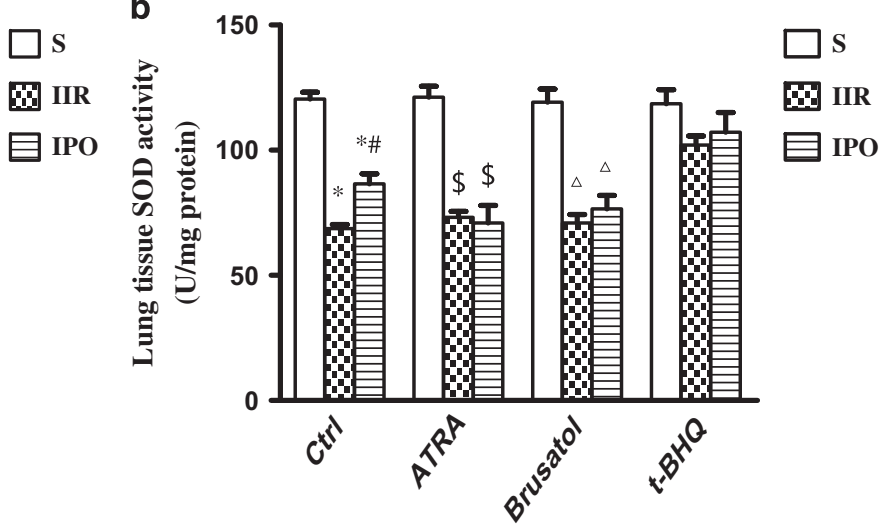

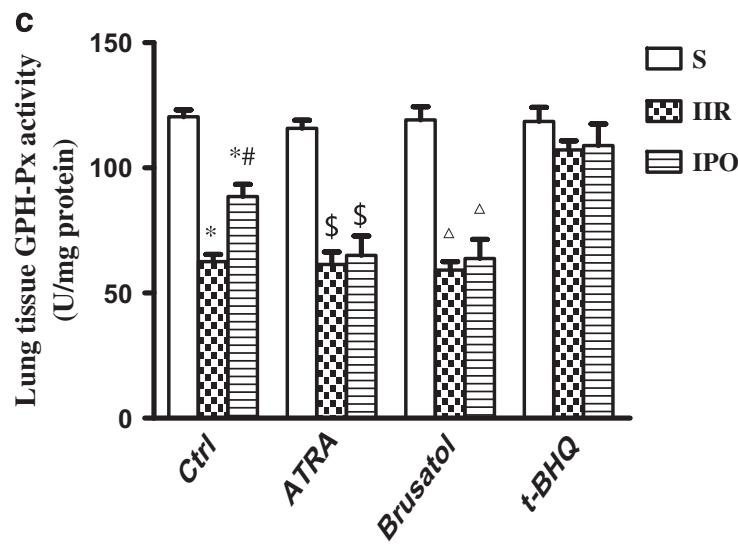

Figure 7 The MDA content and activities of SOD and GSH-PX in lung tissue. The MDA levels (a) as well as the activities of SOD (b) and GSH-Px (c) were determined using colorimetric assays. Data presented as mean \pm s.d., $n=6-10 .{ }^{*} P<0.05$, vs $S$ group, ${ }^{\#} P<0.05$, vs IIR group, ${ }^{5} P<0.05$, vs ATRA+S group, ${ }^{\triangle} P<0.05$, vs Brusatol+S group, $P<0.05$, vs t-BHQ+S group, one-way ANOVA and Tukey's post hoc test.

lipid peroxidation and systemic inflammatory response following IIR. By contrast, Nrf2 inhibitor (ATRA and Brusatol) precluded the beneficial effects of IPo on oxidative stress and systemic inflammatory response. Moreover, t-BHQ (Nrf2 agonist) can have a similar effect as IPo on the oxidative stress and proinflammatory response induced by IIR. All of these results highlight the importance of Nrf2 and HO-1 in the regulation of oxidative stress and proinflammatory response during IIR injury.

In summary, the present study shows that Nrf2 plays a critical role in regulating the HO-1 production induced by IIR. Also, upregulation of Nrf2 expression might represent one of the major mechanisms whereby IPo confers protection against IIR-induced ALI. The beneficial effects of IPo could be attributed to its antioxidative stress and anti-inflammatory properties.

\section{ACKNOWLEDGMENTS}

We thank Dr Na Zhan for her contribution in immunohistochemistry analysis. We thank professor Hong-zhen Hu for his help in writing the manuscript, and also Dr Xian-yu Liu for his helpful discussion of this manuscript. This work was supported by Chinese Natural Science Foundation (No. 81000027, No. 81401574 and No. 81400698).

\section{DISCLOSURE/CONFLICT OF INTEREST}

The authors declare no conflict of interest.

1. Teke Z, Sacar M, Yenisey $C$, et al. Activated protein $C$ attenuates intestinal reperfusion-induced acute lung injury: an experimental study in a rat model. Am J Surg 2008;195:861-873.

2. Mao $Y F$, Zheng XF, Cai JM, et al. Hydrogen-rich saline reduces lung injury induced by intestinal ischemia/reperfusion in rats. Biochem Biophys Res Commun 2009;381:602-605.

3. Swank GM, Deitch EA. Role of the gut in multiple organ failure: bacterial translocation and permeability changes. World J Surg 1996;20:411-417.

4. De Lima FM, Villaverde AB, Albertini R, et al. Dual effect of low-level laser therapy (LLLT) on the acute lung inflammation induced by intestinal ischemia and reperfusion: action on anti- and proinflammatory cytokines. Lasers Surg Med 2011;43:410-420.

5. Zambon $M$, Vincent JL. Mortality rates for patients with acute lung injury/ARDS have decreased over time. Chest 2008;133:1120-1127.

6. Zhao ZQ. Post-conditioning in reperfusion injury: a status report. Cardiovasc Drugs Ther 2010;24:265-279.

7. $\mathrm{Xu} \mathrm{B,} \mathrm{Gao} \mathrm{X,} \mathrm{Xu} \mathrm{J,} \mathrm{et} \mathrm{al.} \mathrm{Ischemic} \mathrm{post-conditioning} \mathrm{attenuates} \mathrm{lung}$ reperfusion injury and reduces systemic proinflammatory cytokine release via heme oxygenase 1. J Surg Res 2011;166:e157-e164.

8. Itoh $\mathrm{K}$, Wakabayashi $\mathrm{N}$, Katoh $\mathrm{Y}$, et al. Keap1 regulates both cytoplasmic- nuclear shuttling and degradation of Nrf2 in response to electrophiles. Genes Cells 2003;8:379-391.

9. Gong P, Stewart D, Hu B, et al. Activation of the mouse heme oxygenase- 1 gene by 15 -deoxy-Delta(12,14)-prostaglandin $\mathrm{J}(2)$ is mediated by the stress response elements and transcription factor Nrf2. Antioxid Redox Signal 2002;4:249-257. 
10. Cho HY, Reddy SP, Kleeberger SR. Nrf2 defends the lung from oxidative stress. Antioxid Redox Signal 2006;8:76-87.

11. Wang XJ, Hayes JD, Henderson CJ, et al. Identification of retinoic acid as an inhibitor of transcription factor Nrf2 through activation of retinoic acid receptor alpha. Proc Natl Acad Sci USA 2007;104:19589-19594.

12. Ren $D$, Villeneuve $N F$, Jiang $T$, et al. Brusatol enhances the efficacy of chemotherapy by inhibiting the Nrf2-mediated defense mechanism. Proc Natl Acad Sci USA 2011;108:1433-1438.

13. Shih AY, Li P, Murphy TH. A small-molecule-inducible Nrf2-mediated antioxidant response provides effective prophylaxis against cerebral ischemia in vivo. J Neurosci 2005;25:10321-10335.

14. Sun $\mathrm{Q}$, Meng $\mathrm{QT}$, Jiang $\mathrm{Y}$, et al. Ginsenoside Rb1 attenuates intestinal ischemia reperfusion induced renal injury by activating Nrf2/ARE pathway. Molecules 2012;17:7195-7205.

15. Liu KX, Li YS, Huang WQ, et al. Immediate but not delayed post-conditioning during reperfusion attenuates acute lung injury induced by intestinal ischemia/reperfusion in rats: comparison with ischemic preconditioning. J Surg Res 2009;157:e55-e62.

16. Nishina K, Mikawa K, Takao Y, et al. ONO-5046 an elastase inhibitor attenuates endotoxininduced acute lung injury in rabbits. Anesth Analg 1997:84:1097-1103.

17. Wu XJ, Xia ZY, Wang LL, et al. Effects of penehyclidine hydrochloride on pulmonary contusion from blunt chest trauma in rats. Injury 2012:43:232-236.

18. Xu XL, Xie QM, Shen $\mathrm{YH}$, et al. Mannose prevents lipopolysaccharideinduced acute lung injury in rats. Inflamm Res 2008;57:104-110.

19. Zhang $\mathrm{X}$, Bedard EL, Potter $\mathrm{R}$, et al. Mitogen-activated protein kinases regulate HO-1 gene transcription after ischemia-reperfusion lung injury. Am J Physiol Lung Cell Mol Physiol 2002;283:L815-L829.

20. Deng $C$, Sun Z, Tong G, et al. $a$-Lipoic acid reduces infarct size and preserves cardiac function in rat myocardial ischemia/reperfusion injury through activation of PI3K/Akt/Nrf2 pathway. PLoS One 2013;8: e58371.

21. JSouza JR, Oliveira RT, Blotta MH, et al. Serum levels of interleukin-6 (IL-6), interleukin-18 (IL-18) and C-reactive protein (CRP) in patients with type-2 diabetes and acute coronary syndrome without ST-segment elevation. Arq Bras Cardiol 2008:90:86-90.

22. Hwang JM, Wang CJ, Chou FP, et al. Inhibitory effect of berberine on tert-butyl hydroperoxide-induced oxidative damage in rat liver. Arch Toxicol 2002;76:664-670.
23. Liu KX, He W, Rinne T, et al. The effect of Ginkgo biloba extract (EGb 761) pretreatment on intestinal epithelial apoptosis induced by intestinal ischemia/ reperfusion in rats: role of ceramide. Am J Chin Med 2007:35:805-819.

24. Garcia-Dorado D, Piper HM. Post-conditioning: reperfusion of "reperfusion injury" after hibernation. Cardiovasc Res 2006;69:1-3.

25. Sola A, Hotter $G$, Prats $N$, et al. Modification of oxidative stress in response to intestinal preconditioning. Transplantation 2000;69: 767-772.

26. Ito $\mathrm{K}$, Ozasa $\mathrm{H}$, Kojima $\mathrm{N}$, et al. Pharmacological preconditioning protects lung injury induced by intestinal ischemia/reperfusion in rat. Shock 2003; 19:462-468.

27. Reddy NM, Kleeberger SR, Kensler TW, et al. Disruption of Nrf2 impairs the resolution of hyperoxia-induced acute lung injury and inflammation in mice. J Immunol 2009;182:7264-7271.

28. Reddy NM, Potteti HR, Mariani TJ, et al. Conditional deletion of Nrf2 in airway epithelium exacerbates acute lung injury and impairs the resolution of inflammation. Am J Respir Cell Mol Biol 2011;45: 1161-1168.

29. Athale J, Ulrich A, MacGarvey NC, et al. Nrf2 promotes alveolar mitochondrial biogenesis and resolution o flung injury in Staphylococcus aureus pneumonia in mice. Free Radic Biol Med 2012;53:1584-1594.

30. Chapple SJ, Siow RC, Mann GE. Crosstalk between Nrf2 and the proteasome: therapeutic potential of Nrf2 inducers in vascular disease and aging. Int J Biochem Cell Biol 2012;44:1315-1320.

31. Otterbein LE, Choi AM. Heme oxygenase: colors of defense against cellular stress. Am J Physiol Lung Cell Mol Physiol 2000;279:L1029-L1037.

32. Carden DL, Granger DN. Pathophysiology of ischaemia-reperfusion injury. J Pathol 2000;190:255-266.

33. Zhang Y, Du Y, Le W, et al. Redox control of the survival of healthy and diseased cells. Antioxid Redox Signal 2011;15:2867-2908.

34. Lapchak PH, loannou A, Kannan L, et al. Platelet-associated CD40/ CD154 mediates remote tissue damage after mesenteric ischemia/ reperfusion injury. PLoS One 2012;7:e32260.

35. Ray PD, Huang BW, Tsuji Y. Reactive oxygen species (ROS) homeostasis and redox regulation in cellular signaling. Cell Signal 2012;24:981-990.

36. Li N, Alam J, Venkatesan Ml, et al. Nrf2 is a key transcription factor that regulates antioxidant defense in macrophages and epithelial cells: protecting against the proinflammatory and oxidizing effects of diesel exhaust chemicals. J Immunol 2004;173:3467-3481. 